\title{
VARIABLE GRID FINITE-DIFFERENCE MODELING INCLUDING SURFACE TOPOGRAPHY
}

\author{
Koichi Hayashi \\ OYO Corporation \\ Technical Research and Development Center \\ 42 Miyukigaoka, Tsukuba \\ Ibaragi, 305-0841 Japan \\ Daniel R. Burns \\ Earth Resources Laboratory \\ Department of Earth, Atmospheric, and Planetary Sciences \\ Massachusetts Institute of Technology \\ Cambridge, MA 02139
}

\begin{abstract}
We have developed a two-dimensional viscoelastic finite-difference modeling method for highly complex topography. Realistic modeling of seismic wave propagation in the near surface is complicated by many factors, such as strong heterogeneity, topographic relief and large attenuation. In order to account for these complications, we use a velocity-stress staggered grid and employ an $O(2,4)$ accurate viscoelastic finite-difference scheme. The implementation includes an irregular free surface condition for topographic relief and a variable grid technique in the shallow parts of the model. Numerical tests indicate that approximately ten grid-points per shortest wavelength results in accurate calculations. The method is accurate and stable, and allows us to handle complex structure in finite-difference modeling.
\end{abstract}

\section{INTRODUCTION}

The demand for investigations of complex geological features is increasing. The seismic method will play a very important role for such investigations, but efficient forward modeling methods are needed. The finite-difference method is one modeling technique that can be used for such complex structures. This method can account for the fac- 


\section{Hayashi and Burns}

tors that complicate seismic wave propagation such as large velocity contrasts, strong heterogeneity, topographic relief, and large attenuation. The modeling of irregular free surface topography and the inclusion of attenuation are two factors that are particularly important in modeling.

Numerical modeling of linear viscoelastic seismic responses in the time domain has recently become practical through algorithms based on the superposition of relaxation mechanisms. These algorithms enable us to calculate attenuating effects inexpensively. For finite-difference modeling of irregular free surfaces, several computation methods have been presented. They can be classified into two main groups. The first is the method in which the finite-difference grid is deformed to match exactly the free surface relief (Hestholm and Ruud, 1994). The second is the method that employs a rectangular grid and generalizes the free surface condition (Robertsson, 1996). The first method is effective for relatively smooth topography, but has limitations for steep topography. On the contrary, the second method has no limitations on the shape of topographic relief. The obvious drawback of the second method is that it requires very fine gridding. Robertsson (1996) found that at least 15 grid-points per wavelength are required. An efficient solution to this dilemma is to use a variable grid approach (De Lilla, 1997) with finer sampling of the grid in the vicinity of the irregular free surface, and a coarse grid in the deeper parts of the model.

In this paper, we will show that the generalized free surface condition combined with the variable grid approach enables us to apply viscoelastic finite-difference modeling to steep and complex structures.

\section{A FREE SURFACE APPROXIMATION IN THE PRESENCE OF TOPOGRAPHY}

Our discussion of the free surface condition and the variable grid method will be concerned with the viscoelastic case. Blanch et al. (1995) presented efficient viscoelastic modeling based on standard linear solids (Appendix A). Robertsson et al. (1994) presented a finite-difference scheme based on the $\mathrm{O}(2,4)$ accurate velocity-stress staggered grid (Levander, 1988) to solve this viscoelastic modeling (Appendix B). We employ this method because the additional memory requirement is small compared with the elastic case. In this method, the stress and strain relaxation times can be calculated by the least square method.

Robertsson (1996) proposed a generalized image method for the free surface condition in which stress-tensor components are imaged and the irregular free surface boundary condition is enforced to ensure that the normal and shear stresses perpendicular to the boundary are zero. This method is based on a robust theoretical derivation and the criteria for stability and accuracy are well established. An alternative method to model surface topography is to let $V p, V s \rightarrow 0$ in the region above the free surface (the density in the region above the free surface is not 0 to avoid numerical instability). This method is called the vacuum formulation (Graves, 1996). This approach is attractive 


\section{Variable Grid Finite-Difference Modeling}

Table 1: Three methods of free-surface approximation

\begin{tabular}{|l|l|l|}
\hline \hline & Calculation of particle velocities & Calculation of stresses \\
\hline Robertsson's generalized & Imaging stresses & $\begin{array}{l}\text { Free surface condition } \\
\text { (the normal and shear } \\
\text { stresses perpendicular to } \\
\text { the boundary are zero). } \\
\text { Set particle velocities to } \\
\text { zero above the free surface. }\end{array}$ \\
\hline Vacuum formulation & No specific calculation & $\begin{array}{l}\text { No specific calculation. } \\
\text { Set P and S wave velocities } \\
\text { to zero above the free surface. }\end{array}$ \\
& & $\begin{array}{l}\text { Free surface condition } \\
\text { (the normal and shear } \\
\text { stresses perpendicular to } \\
\text { the boundary are zero) } \\
\text { Set particle velocities to } \\
\text { zero above the free surface. }\end{array}$ \\
\hline \hline
\end{tabular}

because it can be implemented with the same difference equations used in the interior of the model, and thus, the effects of surface topography are modeled in the same manner as internal media interfaces. It is, however, well known that the method is unstable and inaccurate.

To respect the simplicity of the vacuum method as well as the accuracy of the image method, we propose an accurate and simple condition based on a combination of these approaches. In the proposed method, stresses are calculated so that the normal and shear stresses perpendicular to the boundary are zero, just as in Robertsson's generalized image method. However, the calculation of particle velocities does not involve any specific boundary calculation. Table 1 shows the comparison of the different free surface conditions. Figure 1 shows the staggered finite-difference grid in the vicinity of the free surface used in the proposed method.

To examine the validity of the method, we have carried out a number of numerical tests to compare the three methods (Robertsson's generalized image method, vacuum formulation, and proposed method). Here, we show two examples of the results of numerical tests.

A flat semi-infinite elastic medium was chosen to be the first numerical test for the free surface with topography. This model is similar to the model from a simulation presented by Robertsson (1996). The model is a Poisson solid with P- and S-velocities of 3000 and $1730 \mathrm{~m} / \mathrm{s}$, respectively, and a density of $2500 \mathrm{~kg} / \mathrm{m}^{3}$. In this test, the slope of the surface within the model was varied. If wave propagation is successfully modeled independent of the slope of the surface, then the algorithm should also allow for accurate modeling of a free surface with more general shapes. To avoid problems 


\section{Hayashi and Burns}

due to the uncertainly of the exact location of the source, a P-wave source (15 Hz Ricker wavelet) located $30 \mathrm{~m}$ below the surface was employed. The slope of the free surface was varied from $-90^{\circ}$ to $90^{\circ}$ at $15^{\circ}$ intervals, and the waveforms were collected at $1000 \mathrm{~m}$ source receiver offset in a direction parallel to the free surface. The recorded particle velocity (perpendicular to the free surface) was rotated to the coordinate system of the $0^{\circ}$ case, so that all waveforms can be compared to each other directly. The solution for the $0^{\circ}$ slope can be regard as the exact solution. In Figures 2-4, particle velocity from the simulations with various slopes and their methods are plotted. The receivers were located $50 \mathrm{~m}$ below the surface, and the grid size of $2 \mathrm{~m}$ and $5 \mathrm{~m}$ were used. For the grid size of $2 \mathrm{~m}$ (approximately 50 grid points per wavelength), all methods yield sufficiently accurate results. However, for the grid size of $5 \mathrm{~m}$ (approximately 20 grid points per wavelength), we can see the significant numerical dispersion in the image method and the vacuum method. On the contrary, the dispersion of the proposed method is relatively small.

Figure 5 shows the model used in the second numerical test. The model is an elastic Poisson solid with $\mathrm{P}$ - and S-velocities of 2000 and $1155 \mathrm{~m} / \mathrm{s}$, respectively, and a density of $1000 \mathrm{~kg} / \mathrm{m}^{3}$. A P-wave source (110 Hz Ricker wavelet) located at a distance of $30 \mathrm{~m}$ and $5 \mathrm{~m}$ below the surface was employed. In Figure 6, particle velocity (vertical component) from the simulations with the various grid sizes is plotted. The receivers were located at a distance of $120 \mathrm{~m}$ and $4 \mathrm{~m}$ below the surface. For a large number of grid-points per wavelength ( 40 or 80 ), all methods yield sufficiently accurate results. However, in the case of a smaller number of grid points (10 or 20), we can see that the proposed method is most accurate.

These results imply that the proposed method is most accurate, and at least 20 to 40 grid-points per wavelength is required.

\section{INVESTIGATION OF STAIR-SHAPED BOUNDARY}

Robertsson (1996) mentioned that the generalized free surface condition that we employed yields a good representation of a "staircase-shaped" function, whereas a smooth boundary must be discretized in terms of such steps. Fuyuki and Matsumoto (1980) found that the scattering of Rayleigh waves can be substantial from relatively small steps compared to the wavelength. This scattering from the stair-shaped boundary should be avoided when we model a smooth boundary. In addition, our free surface numerical tests did not reveal whether the observed Rayleigh wave dispersion was an actual wave phenomenon or a numerical error. Thus, we performed the following tests to reveal the effect of stair-shaped discretization on a smooth free surface.

Figure 7 shows the $45^{\circ}$ slope model used in the numerical test. The model is an elastic solid with P- and S-velocities of 5082 and $3000 \mathrm{~m} / \mathrm{s}$, respectively, and a density of $2000 \mathrm{~kg} / \mathrm{m}^{3}$. A P-wave source ( $200 \mathrm{~Hz}$ Ricker wavelet) located at a distance of $25 \mathrm{~m}$ and $6 \mathrm{~m}$ below the surface was employed. In this test, not only the grid size but also the step size of the slope was varied. The step size and the grid size are defined in Figure 8. 


\section{Variable Grid Finite-Difference Modeling}

Table 2: Grid and step sizes and the resulting dispersion.

\begin{tabular}{|c|c|c|c|c|c|c|}
\hline \hline Model & $\begin{array}{c}\text { Grid size } \\
(\mathrm{m})\end{array}$ & $\begin{array}{c}\text { Step size } \\
(\mathrm{m})\end{array}$ & $\begin{array}{c}\text { Wavelength/ } \\
\text { grid size }\end{array}$ & $\begin{array}{c}\text { Wavelength/ } \\
\text { step size }\end{array}$ & Dispersion & Figure \\
\hline $\mathrm{A}$ & 0.5 & 0.5 & 30 & 30 & No & Figure 9a \\
\hline $\mathrm{B}$ & 0.5 & 1.0 & 30 & 15 & No & Figure 9b \\
\hline $\mathrm{C}$ & 1.0 & 1.0 & 15 & 15 & No & Figure 9c \\
\hline $\mathrm{D}$ & 0.5 & 1.5 & 30 & 10 & No & Figure 9d \\
\hline $\mathrm{E}$ & 1.5 & 1.5 & 10 & 10 & Small & Figure 9e \\
\hline $\mathrm{F}$ & 0.5 & 2.0 & 30 & 7.5 & Small & Figure 9f \\
\hline $\mathrm{G}$ & 2.0 & 2.0 & 7.5 & 7.5 & Medium & Figure 9g \\
\hline $\mathrm{H}$ & 0.5 & 3.0 & 30 & 5 & Medium & Figure 9h \\
\hline $\mathrm{I}$ & 3.0 & 3.0 & 5 & 5 & Large & Figure 9i \\
\hline \hline
\end{tabular}

In Figure 9, particle velocity (vertical component) from the simulations with the various grid and step sizes is plotted. The receivers were located $4 \mathrm{~m}$ below the surface and at $6 \mathrm{~m}$ distance intervals. Table 2 shows the list of the grid and step sizes as well as the results of the computation.

The results of the computation can be summarized as follows. The results from model $\mathrm{A}$ to $\mathrm{C}$ (Figures $9 \mathrm{a}-\mathrm{c}$ ) have no large difference and no dispersion; we can consider these waveforms to be accurate. Model D (Figure 9d) and model E (Figure 9e) are the same step size, however, the dispersion of model $\mathrm{E}$ is larger than that of model $\mathrm{D}$. We can see a similar tendency in model F, G (Figures $9 \mathrm{f}$ and $9 \mathrm{~g}$ ) and model H, I (Figure $9 \mathrm{~h}$ and $9 \mathrm{i}$ ) in which the step sizes are equivalent. This result implies that the numerical errors due to large grid sizes are larger than the Rayleigh wave scattering due to the stair-shaped boundary.

The above numerical tests were performed on a model with a constant slope of $45^{\circ}$. However, we can expect that the constant slope is accurate compared to the surface with arbitrary slope. To confirm that the accuracy does not depend on the curvature of the free surface boundary, we performed another numerical test using the model shown in Figure 10. In this test, the free surface boundary is curved smoothly. The model is an elastic solid with P- and S-velocities of 6000 and $3000 \mathrm{~m} / \mathrm{s}$, respectively, and a density of $1800 \mathrm{~kg} / \mathrm{m}^{3}$. A P-wave source (100 Hz Ricker wavelet) located at a distance of $24 \mathrm{~m}$ and $12 \mathrm{~m}$ below the surface was employed. In this test, the grid sizes were set to $0.5 \mathrm{~m}, 1.0 \mathrm{~m}, 2.0 \mathrm{~m}$ and $3.0 \mathrm{~m}$, and the shortest wavelength is approximately $30 \mathrm{~m}$ (corresponding to grid-points per wavelength of $60,30,15$ and 10 , respectively). The grid size and step size are equivalent in this test. In Figure 11, particle velocity (vertical component) from the simulations with the various grid sizes is plotted. The waveforms were obtained at receivers located $12 \mathrm{~m}$ below the surface and at $12 \mathrm{~m}$ distance intervals. With 60 grid-points per wave length (Figure 11a), there is no dispersion and it can be considered as accurate. As the grid size increases, the dispersion due to the stair-shaped surface boundary increases. However, we can see that the dispersion is mainly generated 


\section{Hayashi and Burns}

at the distance between 200 and $250 \mathrm{~m}$ where the slope of surface is relatively gentle. To compare this result with the constant $45^{\circ}$ case mentioned before, we can see that the dispersion of the latter is larger than the former.

These results imply that the proposed method requires at least 30 grid-points per wavelength. In particular, relatively gentle slopes require a large number of grid points per wavelength. The cause of the dispersion is mainly a numerical error due to the large grid sizes rather than the actual Rayleigh wave scattering due to the stair-shaped free surface boundary.

\section{VARIABLE GRID FINITE-DIFFERENCE METHOD}

A uniformly-spaced grid used in a model containing an irregular surface and low velocities in the shallow subsurface would require large portions of the model to be strongly over sampled. The resulting memory requirements would severely limit the size of the models. An obvious solution to this dilemma is to use a finer sampling of the grid in the vicinity of the free surface compared to the deeper parts of the model. The variable grid approach allows us to vary the discretization of the model and the wavefield with respect to the velocity structure. Compared to a standard uniform finite-difference grid approach, this method saves a considerable amount of memory and computations. Therefore, we combined the variable grid method with our irregular free-surface modeling.

Figure 12 shows the staggered finite-difference grid in the vicinity of the boundary between different grid sizes. We use a three times finer grid (both horizontally and vertically) in the near surface or low velocity region compared to the rest of the model. This implementation allows us to avoid any limitation of the shape of the grid size boundary. Figure 13 shows the computation procedure of the proposed variable grid method. An example of the snapshot of the variable grid calculation is shown in Figure 14. We can see that artificial reflections from the boundary between the different grid sizes is sufficiently small.

However, the simple implementation of this variable grid method is not stable if we perform a large number of time steps. Figure 15 shows an example of the instability due to the variable grid computation. In order to reduce the instability of the computation, we applied averaging or weighting to the replacement of the coarse grid components within the fine grid. Figure 16 shows several methods of averaging or weighting applied to the replacement components. Figure 17 shows the energy of the waveforms shown in Figure 15 integrated over time with various replacement methods. As a result of this comparison, we conclude that 5 point averaging or 9 point weighting is most stable. This result implies that the weight of the center node of the stencil is crucial for a stable computation.

We applied the variable grid method to the calculation of irregular topography mentioned above. The area of the fine grid is shown in Figure 18. The calculated waveforms by the proposed method with the various grid sizes are shown in Figure 19. 


\section{Variable Grid Finite-Difference Modeling}

The result shows that a coarse grid size of $1 \mathrm{~m}$ combined with a fine grid size of $1 / 3 \mathrm{~m}$ gives a more accurate solution than using a $0.5 \mathrm{~m}$ uniform grid. This result suggests that approximately ten grid-points per wavelength, combined with the variable grid method, will give an accurate solution to a model with an irregular surface.

\section{CONCLUSIONS}

An accurate and simple irregular free surface condition and a stable variable grid finitedifference approximation are proposed. These two techniques can be applied to irregular free surface calculations simultaneously. The new method is simple to implement in conventional staggered grid finite-difference schemes, is computationally efficient, and enables modeling of highly irregular topography. It can be seen that approximately ten grid-points per wavelength ensures accuracy in the calculation of an irregular surface when combined with a variable grid implementation.

\section{ACKNOWLEDGMENTS}

We wish to thank Johan O. A. Robertsson, Matthias G. Imhof, Roque Szeto, and M. Nafi Toksöz for many discussions and helpful suggestions.

This work was supported by the Borehole Acoustics and Logging/Reservoir Delineation Consortia at the Massachusetts Institute of Technology, and by the Department of Energy grant \#DE-FG02-98ER14846. 


\section{Hayashi and Burns}

\section{REFERENCES}

Blanch, J.O., Robertsson, J.O., and Symes, W.W., 1995, Modeling of a constant Q: Methodology and algorithm for an efficient and optimally inexpensive viscoelastic technique, Geophysics, 60, 176-184.

De Lilla, A., 1997, Finite difference seismic wave propagation using variable grid sizes, M.Sc. Thesis, Massachusetts Institute of Technology.

Fuyuki, M., and Matsumoto, Y., 1980, Finite-difference analysis of Rayleigh wave scattering at a trench, Bull. Seismol. Soc. Am., 86, 1091-1106.

Graves, R.W., 1996, Simulating seismic wave propagation in 3D elastic media using staggered-grid finite differences, Bull. Seismol. Soc. Am., 86, 1091-1106.

Hestholm, S.O. and Ruud, B.O., 1994, 2D finite-difference elastic wave modeling including surface topography, Geophys. Prosp., 42, 371-390.

Levander, A.R., 1988, Fourth-order finite-difference P-SV seismograms, Geophysics, 53, $1425-1436$.

Robertsson, J.O., 1996, A numerical free-surface condition for elastic/viscoelastic finitedifference modeling in the presence of topography, Geophysics, 61, 1921-1934.

Robertsson, J.O., Blanch, J.O., and Symes, W.W., 1994, Viscoelastic finite-difference, Geophysics, 59, 1444-1456. 


\section{Variable Grid Finite-Difference Modeling}

\section{Appendix A. VISCOELASTIC MODELING USING $\tau$}

This appendix will briefly describe the $\tau$-method for the viscoelastic modeling proposed by Blanch et al. (1995). The SLS (a spring and dashpot in series, in parallel with a spring) has been shown to be a general mechanical viscoelastic model. An array of L-SLS has the stress relaxation function.

$$
G(t)=M_{R}\left(1-\sum_{l=1}^{L}\left(1-\frac{\tau_{\varepsilon l}}{\tau_{\sigma l}}\right) e^{\frac{-t}{\tau_{\sigma l}}}\right) \theta(t)
$$

where $\theta(t)$ is the Heaviside function, $M_{R}$ is the relaxed stress modulus corresponding to $G(t)$, and $\tau_{\sigma l}$ and $\tau_{\varepsilon l}$ are the stress and strain relaxation times for the $l$ th SLS.

The complex stress modulus $M_{C}(\omega)$ is defined as the Fourier transform of the stress relaxation function. The quality factor $Q$ is defined as,

$$
Q(\omega)=\frac{\operatorname{Re}(M c(\omega))}{\operatorname{Im}(M c(\omega))}
$$

This equation defines $Q$ as the number of wavelengths a pulse may propagate before its amplitude drops by a factor of $e^{-\pi}$. Thus, $Q$ is a function of frequency. For an array of standard linear solids, (A-1) and (A-2) yields,

$$
Q(\omega)=\frac{1-L+\sum_{l=1}^{L} \frac{1+\omega^{2} \tau_{c l} \tau_{\sigma l}}{1+\omega^{2} \tau_{\sigma l}^{2}}}{\sum_{l=1}^{L} \frac{\omega\left(\tau_{\varepsilon l}-\tau_{\sigma l}\right)}{1+\omega^{2} \tau_{\sigma l}^{2}}} .
$$

The $\tau$ method is based on the simple observation that the level of attenuation caused by an SLS can be determined by a dimensionless (frequency scale independent) variable $\tau$. If we defined $\tau$ as,

$$
\tau=\frac{\tau_{\varepsilon l}}{\tau_{\sigma l}}-1=\frac{\tau_{\varepsilon l}-\tau_{\sigma l}}{\tau_{\sigma l}}
$$

the inverse of $Q$ for one SLS can be written as,

$$
Q^{-1}=\frac{\omega \tau_{\varepsilon l} \tau}{1+\omega^{2} \tau_{\sigma l}^{2}(1+\tau)}
$$

Using the parameter $\tau$ to tune an array of SLS's, and assuming that $1+\tau \approx 1$, equation $(\mathrm{A}-3)$ yields,

$$
Q^{-1}=\sum_{l=1}^{L} \frac{\omega \tau_{\varepsilon l} \tau}{1+\omega^{2} \tau_{\sigma l}^{2}} .
$$

In this expression $Q^{-1}$ is linear in $\tau$. Therefore, we can easily find the best approximation in the least square sense over a predefined frequency range to any $Q_{0}$ by minimizing over $\tau$ the expression,

$$
J=\int_{\omega_{a}}^{\omega_{b}}\left(Q^{-1}\left(\omega, \tau, \tau_{l}\right)-Q_{0}^{-1}\right)^{2} d \omega
$$




\section{Hayashi and Burns}

to zero and solve for $\tau$. To find the minimum, we set the derivative of $J$ with respect to $\tau$ to zero and solve for $\tau$.

$$
\frac{d J}{d \tau}=2 \int_{\omega_{a}}^{\omega_{b}}\left(Q^{-1}\left(\omega, \tau, \tau_{l}\right)-Q_{0}^{-1} \times \frac{d Q^{-1}\left(\omega, \tau_{\sigma l}, \tau\right)}{d \tau} d \omega=0 .\right.
$$

The final formula for $\tau$ is

$$
\tau=\frac{1}{Q_{0}} \cdot \frac{\sum_{l=1}^{L} I_{0 l}}{\sum_{l=1}^{L} I_{1 l}+2 \sum_{l=1}^{L-1} \sum_{k=l+1}^{L} I_{2 k l}}
$$

where,

$$
\begin{aligned}
I_{0 l} & =\frac{1}{2 \tau_{\sigma l}}\left[\log \left(1+\omega^{2} \tau_{\sigma l}^{2}\right)\right]_{\omega_{a}}^{\omega_{b}} \\
I_{1 l} & =\frac{1}{2 \tau_{\sigma l}}\left[\arctan \left(\omega \tau_{\sigma l}\right)-\frac{\omega \tau_{\sigma l}}{1+\omega^{2} \tau_{\sigma l}^{2}}\right]_{\omega_{a}}^{\omega_{b}} \\
I_{2 l k} & =\frac{\tau_{\sigma l} \tau_{\sigma k}}{\tau_{\sigma k}^{2}-\tau_{\sigma l}^{2}}\left[\frac{\arctan \left(\omega \tau_{\sigma l}\right)}{\tau_{\sigma l}}-\frac{\arctan \left(\omega \tau_{\sigma k}\right)}{\tau_{\sigma k}}\right]_{\omega_{a}}^{\omega_{b}} \\
\tau_{\sigma l} & =\frac{1}{\omega_{l}} .
\end{aligned}
$$




\section{Variable Grid Finite-Difference Modeling}

\section{Appendix B. VISCOELASTIC STAGGERED GRID FINITE-DIFFERENCE METHOD}

This appendix briefly describes the viscoelastic finite-difference modeling proposed by Robertsson et al. (1994). In 2-D viscoelastic staggered grid finite-difference modeling, the following three sets of equations are solved.

Equations governing stress:

$$
\begin{aligned}
\frac{\partial \sigma_{x x}}{\partial t} & =\pi \frac{\tau_{\varepsilon}^{p}}{\tau_{\sigma}}\left(\frac{\partial \nu_{x}}{\partial x}+\frac{\partial \nu_{z}}{\partial z}\right)-2 \mu \frac{\tau_{\varepsilon}^{s}}{\tau_{\sigma}} \frac{\partial \nu_{z}}{\partial z}+r_{x x} \\
\frac{\partial \sigma_{z z}}{\partial t} & =\pi \frac{\tau_{\varepsilon}^{p}}{\tau_{\sigma}}\left(\frac{\partial \nu_{x}}{\partial x}+\frac{\partial \nu_{z}}{\partial z}\right)-2 \mu \frac{\tau_{\varepsilon}^{s}}{\tau_{\sigma}} \frac{\partial \nu_{x}}{\partial x}+r_{z z} \\
\frac{\partial \sigma_{x z}}{\partial t} & =\mu \frac{\tau_{\varepsilon}^{s}}{\tau_{\sigma}}\left(\frac{\partial \nu_{x}}{\partial z}+\frac{\partial \nu_{z}}{\partial x}\right)+r_{x z} .
\end{aligned}
$$

Equations governing particle velocities:

$$
\begin{aligned}
& \frac{\partial \nu_{x}}{\partial t}=\frac{1}{\rho}\left(\frac{\partial \sigma_{x x}}{\partial x}+\frac{\partial \sigma_{x z}}{\partial z}\right) \\
& \frac{\partial \nu_{z}}{\partial t}=\frac{1}{\rho}\left(\frac{\partial \sigma_{x z}}{\partial x}+\frac{\partial \sigma_{z z}}{\partial z}\right) .
\end{aligned}
$$

Equations governing the so-called memory variables, which are introduced to eliminate the numerically inconvenient convolution arising in the viscoelastic constitutive relation:

$$
\begin{aligned}
\frac{\partial r_{x x}}{\partial t} & =-\frac{1}{\tau_{\sigma}}\left(r_{x x}+\pi\left(\frac{\tau_{\varepsilon}^{p}}{\tau_{\sigma}}-1\right)\left(\frac{\partial \nu_{x}}{\partial x}+\frac{\partial \nu_{z}}{\partial z}\right)-2 \mu\left(\frac{\tau_{\varepsilon}^{s}}{\tau_{\sigma}}-1\right) \frac{\partial \nu_{z}}{\partial z}\right) \\
\frac{\partial r_{z z}}{\partial t} & =-\frac{1}{\tau_{\sigma}}\left(r_{z z}+\pi\left(\frac{\tau_{\varepsilon}^{p}}{\tau_{\sigma}}-1\right)\left(\frac{\partial \nu_{x}}{\partial x}+\frac{\partial \nu_{z}}{\partial z}\right)-2 \mu\left(\frac{\tau_{\varepsilon}^{s}}{\tau_{\sigma}}-1\right) \frac{\partial \nu_{x}}{\partial x}\right) \\
\frac{\partial r_{x z}}{\partial t} & =-\frac{1}{\tau_{\sigma}}\left(r_{x z}+\mu\left(\frac{\tau_{\varepsilon}^{s}}{\tau_{\sigma}}-1\right)\left(\frac{\partial \nu_{x}}{\partial z}+\frac{\partial \nu_{z}}{\partial x}\right)\right) .
\end{aligned}
$$

$\sigma_{i j}$ : the $i j$ th component of the symmetric stress tensor.

$\nu_{i}$ : the $i$ th component of the particle velocity.

$r_{i j}$ : the memory variables.

$\tau_{\varepsilon}^{p}, \tau_{\varepsilon}^{s}$ : the viscoelastic strain relaxation times for $\mathrm{P}$ - and $\mathrm{SV}$-waves, respectively. $\tau_{\sigma}$ : the viscoelastic stress relaxation time for both the P-and SV waves.

$\mu$ : the relaxation modulus corresponding to $\mathrm{SV}$-waves, which is analogous to Lamé constant $\mu$ in the elastic case.

$\pi$ : the relaxation modulus corresponding to $\mathrm{P}$-waves, which is analogous to $\lambda+2 \mu$ in the elastic case. 


\section{Hayashi and Burns}

$\rho$ : the density.

In the 2-D case, the viscoelastic horizontal free-surface satisfies the following conditions:

$$
\begin{aligned}
\sigma_{i z} & =0 \\
r_{i z} & =0 .
\end{aligned}
$$

These conditions lead the following equations by the use of equations (B-1) to (B-3):

$$
\begin{aligned}
& \frac{\partial \nu_{x}}{\partial z}=-\frac{\partial \nu_{z}}{\partial x} \\
& \frac{\partial \nu_{z}}{\partial z}=-\left(1-2 \frac{\tau_{\varepsilon}^{s}}{\tau_{\varepsilon}^{p}} \frac{\mu}{\pi}\right) \frac{\partial \nu_{x}}{\partial x} .
\end{aligned}
$$

Vertical free-surface boundaries can be implemented in the same way. 


\section{Variable Grid Finite-Difference Modeling}

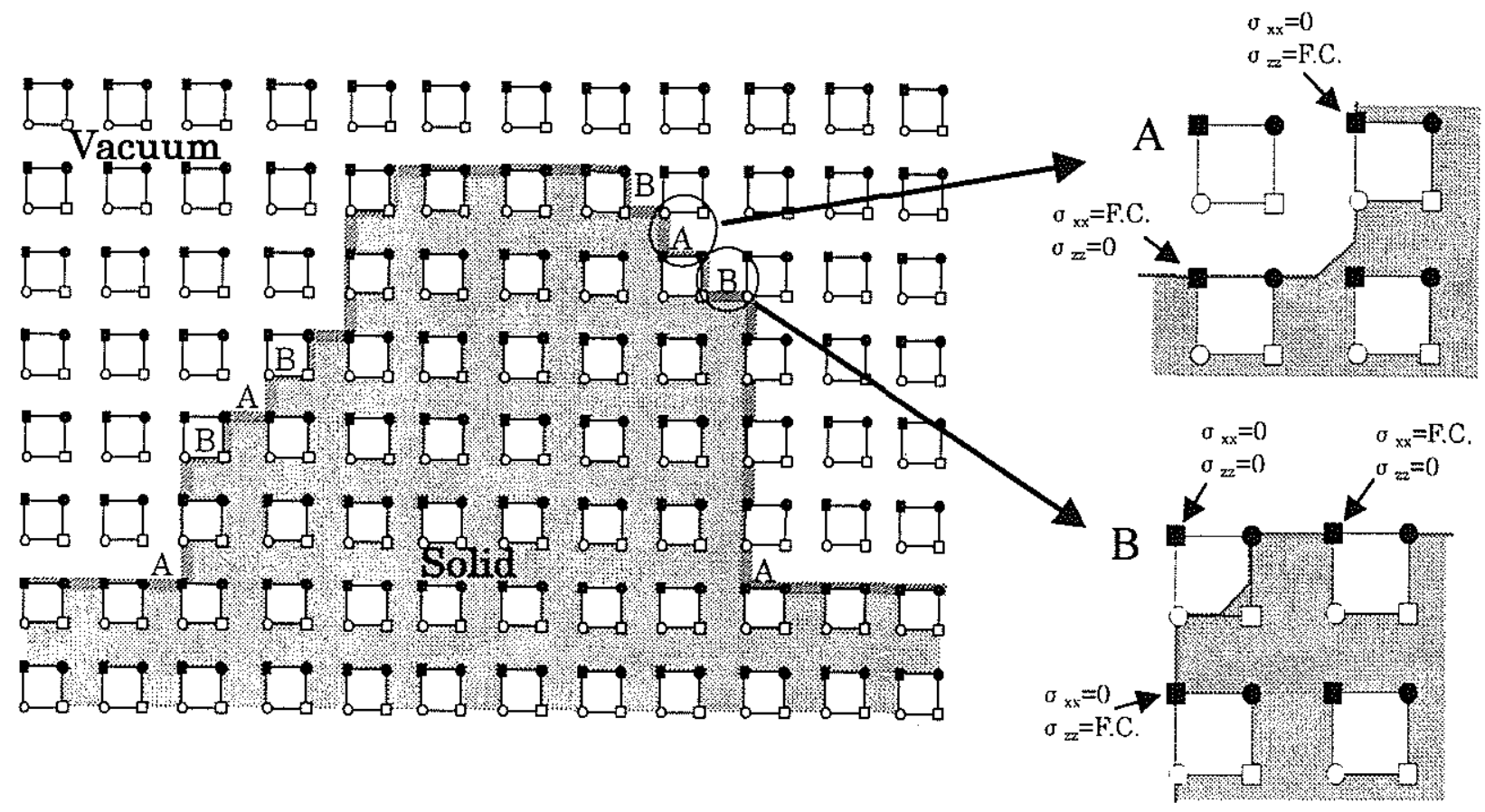

Figure 1: Staggered finite-difference grid in the vicinity of free surface boundary. On the boundary, free surface condition (F.C.) is that the normal and shear stresses perpendicular to the boundary are zero. Within the grid-cells, the solid squares represent the $\sigma_{x x}, \sigma_{z z}, r_{x x}, r_{z z}$ components; the light squares the $\sigma_{x z}, r_{x z}$ components; the solid circles the $v_{x}$ components; and the light circles represent the $v_{z}$ components ( $\sigma$ : stress; $r$ : memory variable; $v$ : velocity). 


\section{Hayashi and Burns}

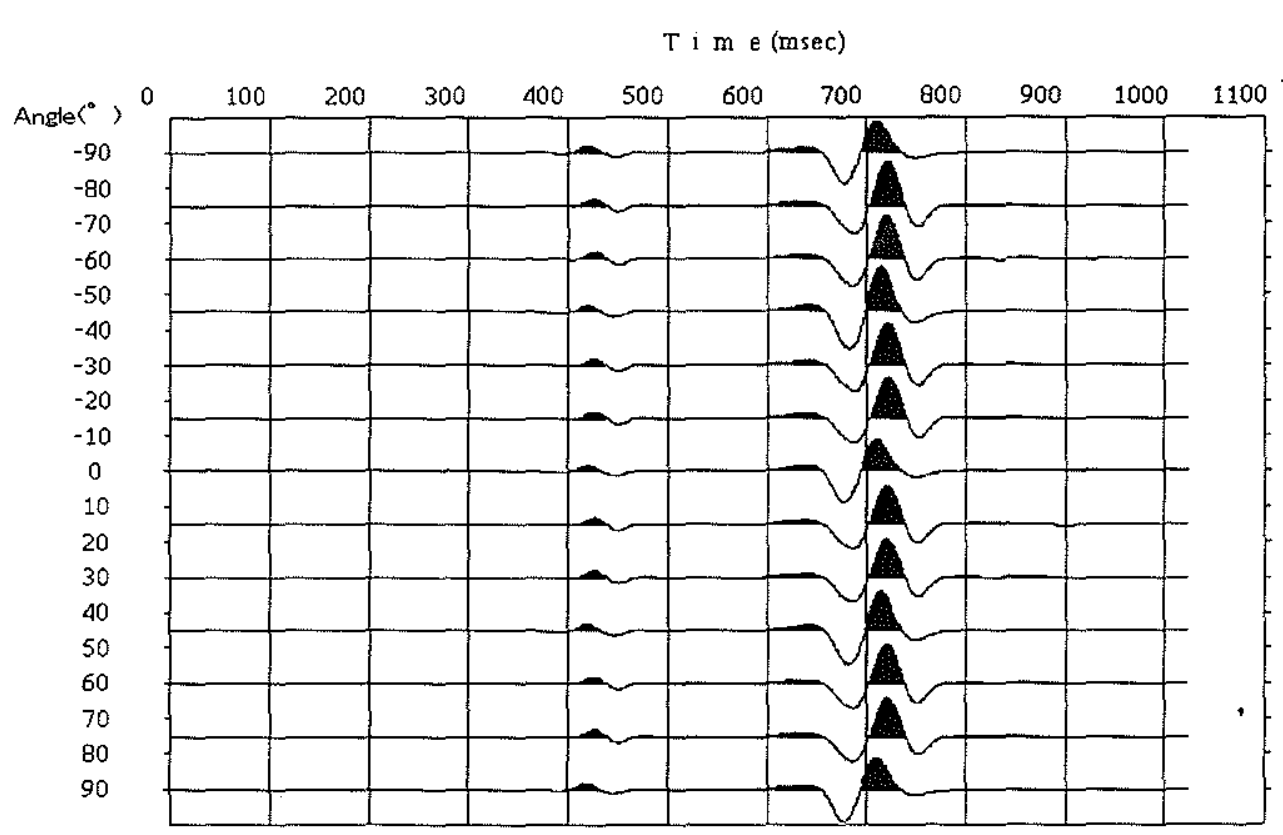

(a) Image method. Grid size $=2 \mathrm{~m}(\lambda / \Delta \mathrm{x}=50)$.

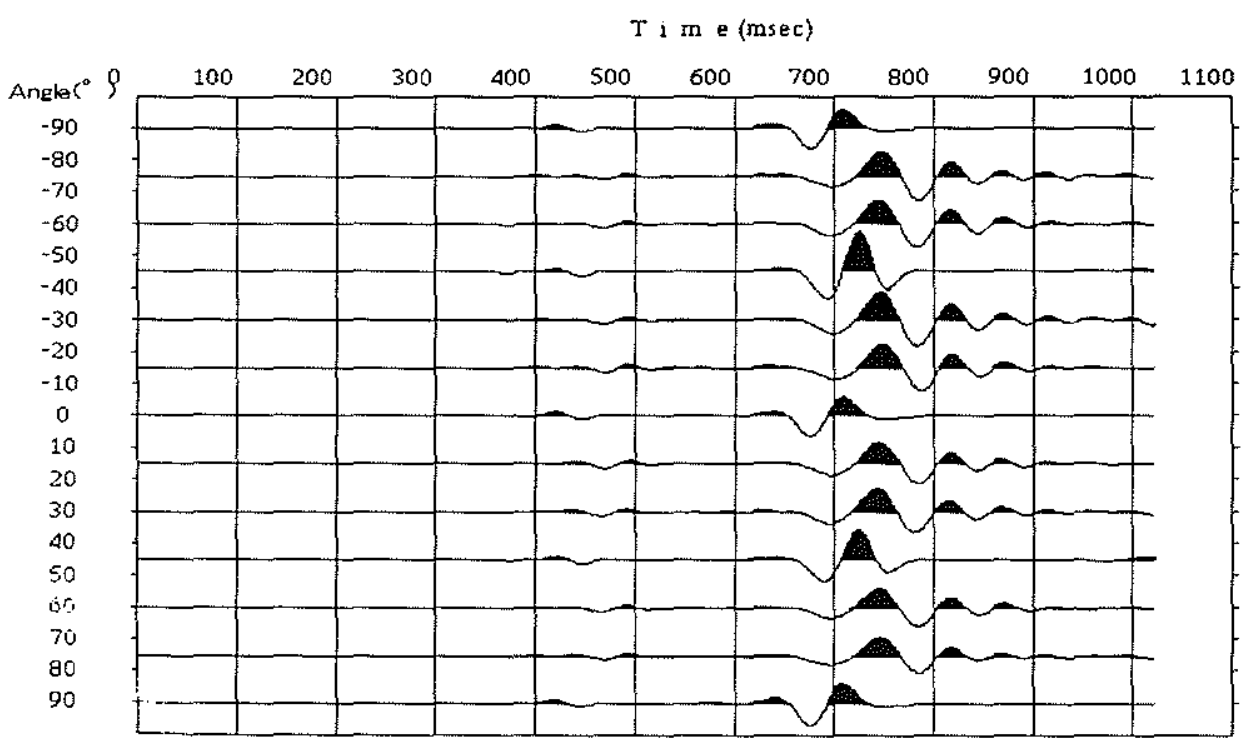

(b) Image method. Grid size $=5 \mathrm{~m}(\lambda / \Delta \mathrm{x}=20)$.

Figure 2: Image method. Time series collected at $50 \mathrm{~m}$ below the free surface and $100 \mathrm{~m}$ source-receiver offset. Particle velocity perpendicular to the free surface is plotted. The angles correspond to the dip of the flat free surface $\left(0^{\circ}\right.$ is horizontal). (a) Grid size $=2 \mathrm{~m}(\lambda$ (wavelength $) / \Delta x($ grid size $)=50)$. (b) Grid size $=5 \mathrm{~m}(\lambda / \Delta x=20)$. 
Variable Grid Finite-Difference Modeling

$\mathrm{T}$ i $\mathrm{m} \mathrm{e}$ (msec)

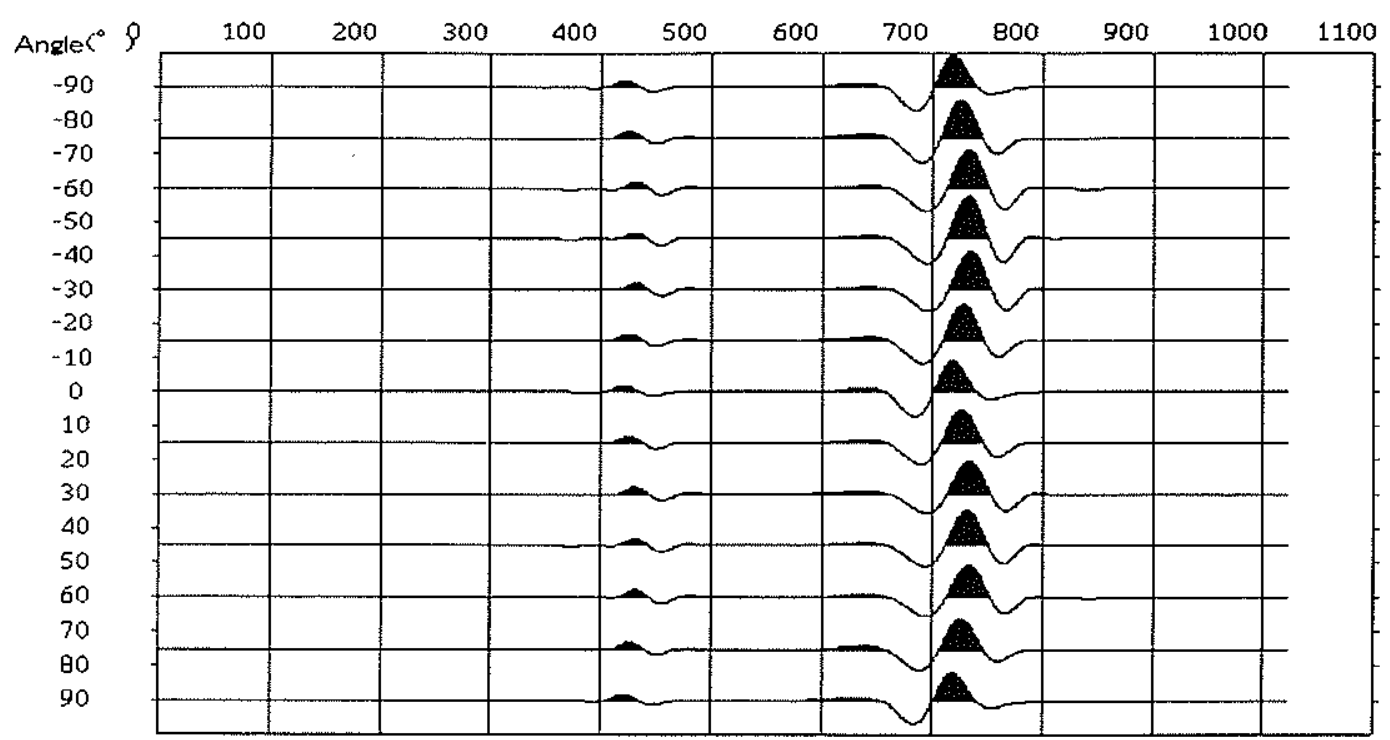

(a) Vacuum formulation. Grid size $=2 \mathrm{~m}(\lambda / \Delta \mathrm{x}=50)$.

T I $\mathrm{m} e$ (msec)

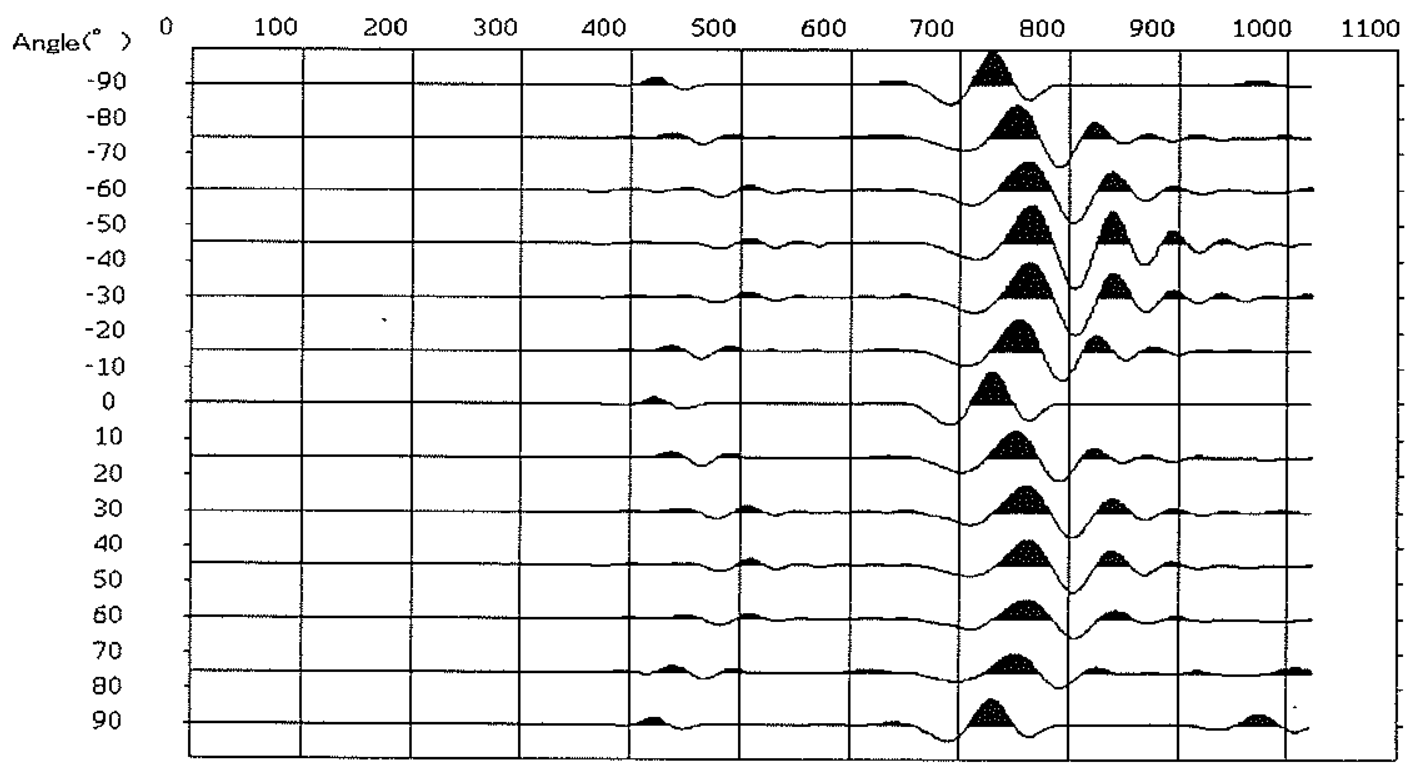

(b) Vacuum formulation. Grid size $=5 \mathrm{~m}(\lambda / \Delta \mathrm{x}=20)$.

Figure 3: Vacuum formulation. Time series collected at $50 \mathrm{~m}$ below the free surface and $1000 \mathrm{~m}$ source-receiver offset. Particle velocity perpendicular to the free surface is plotted. The angles correspond to the dip of the flat free surface $\left(0^{\circ}\right.$ is horizontal). (a) Grid size $=2 \mathrm{~m}(\lambda / \Delta x=50)$. (b) Grid size $=5 \mathrm{~m}(\lambda / \Delta x=20)$. 


\section{Hayashi and Burns}

$\mathrm{T} 1 \mathrm{~m} \in$ (msec)

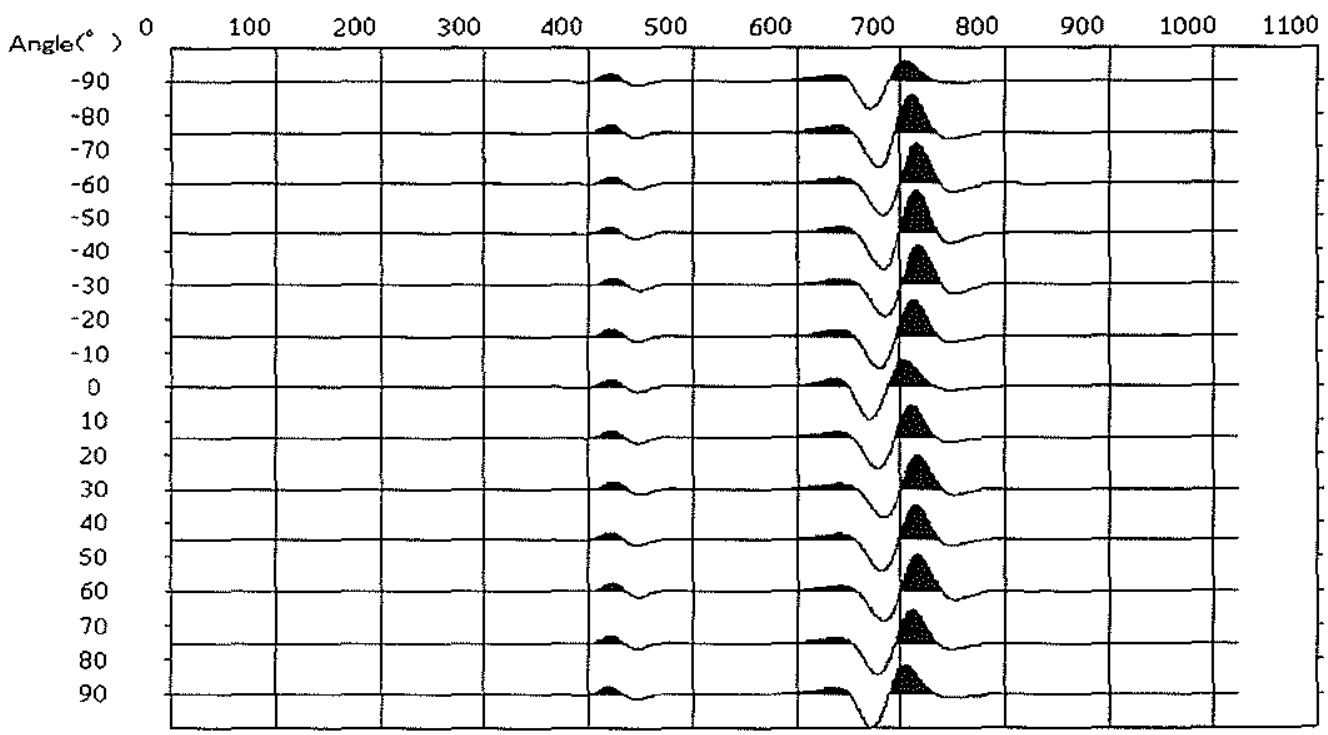

(a) Proposed method. Grid size $=2 \mathrm{~m}(\lambda / \Delta \mathrm{x}=50)$.

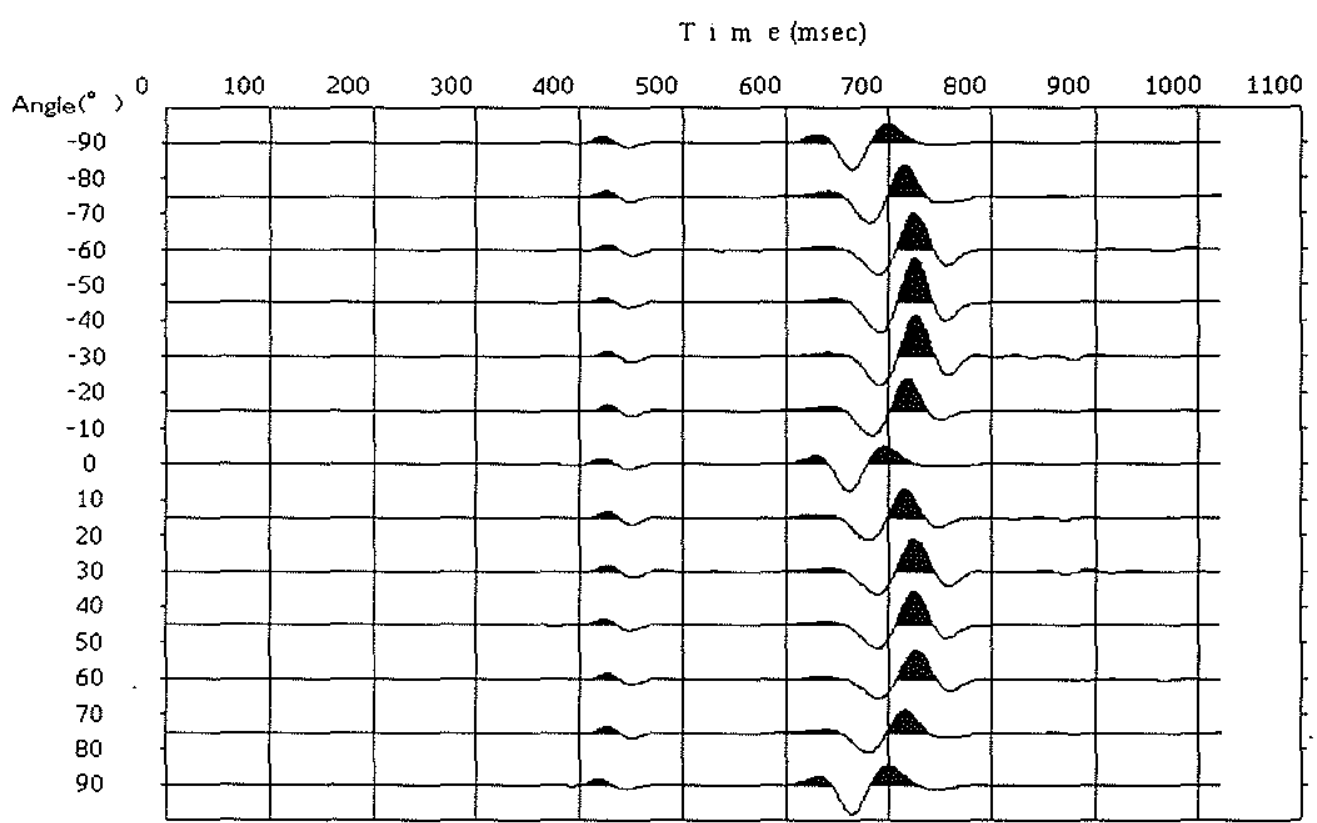

(b) Proposed method. Grid size $=5 \mathrm{~m}(\lambda / \Delta \mathrm{x}=20)$.

Figure 4: Proposed method. Time series collected at $50 \mathrm{~m}$ below the free surface and $1000 \mathrm{~m}$ source-receiver offset. Particle velocity perpendicular to the free surface is plotted. The angles correspond to the dip of the flat free surface $\left(0^{\circ}\right.$ is horizontal). (a) Grid size $=2 \mathrm{~m}(\lambda / \Delta x=50)$. (b) Grid size $=5 \mathrm{~m}(\lambda / \Delta x=20)$. 


\section{Variable Grid Finite-Difference Modeling}

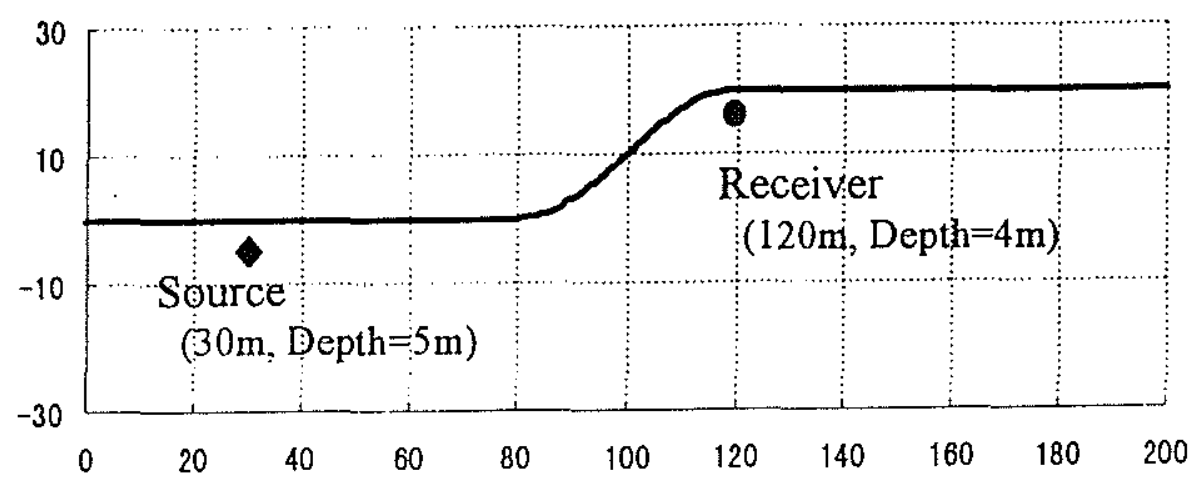

Figure 5: Model used in the second numerical test. P-velocity $=2000 \mathrm{~m} / \mathrm{s}$;

S-velocity $=1155 \mathrm{~m} / \mathrm{s} ;$ density $=1000 \mathrm{~kg} / \mathrm{m}^{3} ; \mathrm{Qp}=10000 ; \mathrm{Qs}=10000 ;$ Sourcer $=$ Ricker wavelet $(110 \mathrm{~Hz})$. Minimum wavelength (S-wave) is approximately $10 \mathrm{~m}$. 


\section{Hayashi and Burns}

T i m e (msec)

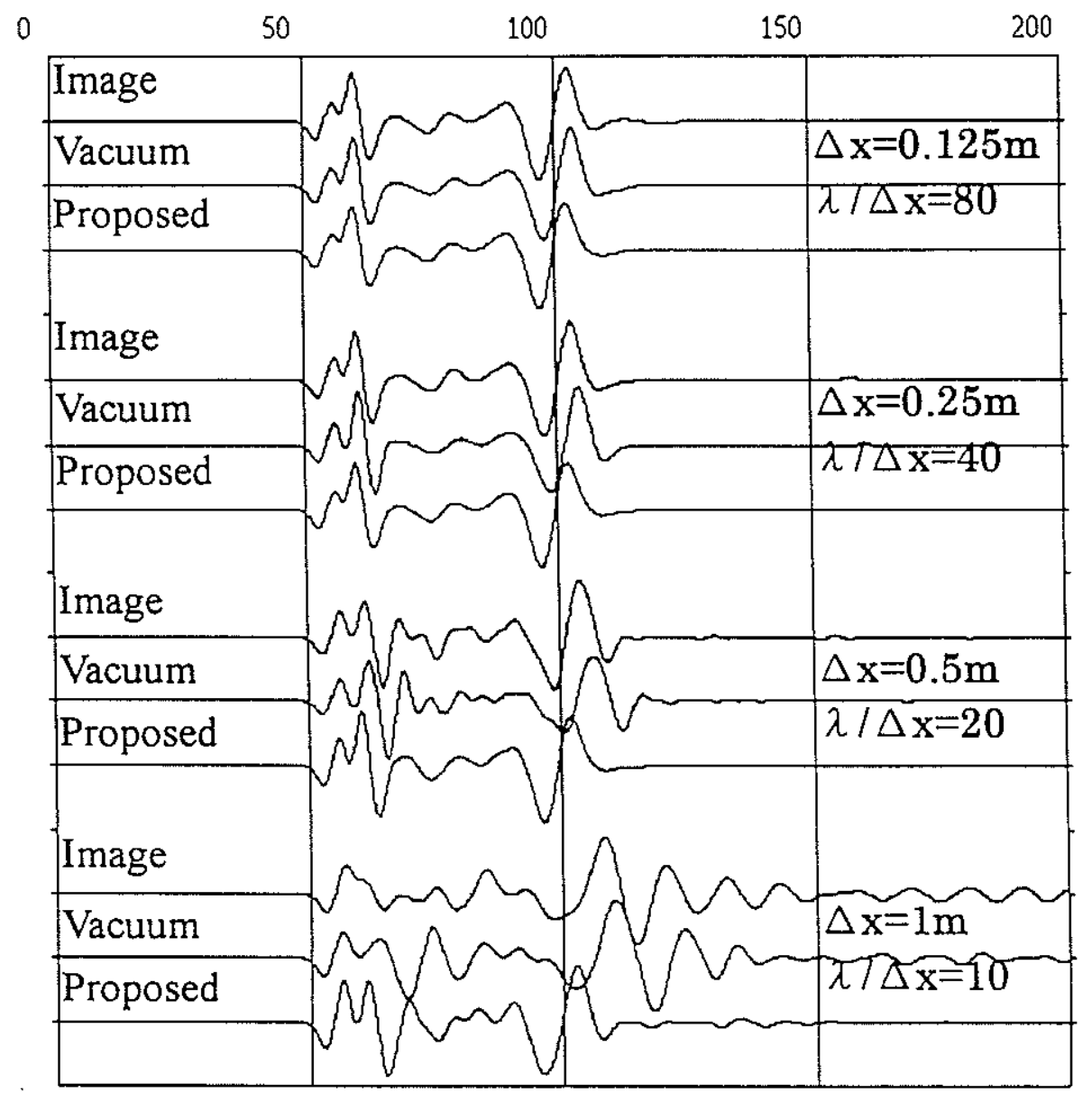

Figure 6: Time series of particl velocity perpendicular to the free surface with various grid sizes for the model given in Figure 5. $\Delta x$ : grid size; $\lambda$ : minimum wavelength (approximately $10 \mathrm{~m}$ ). Image: Robertsson's generalized image method. Vacuum: Vacuum formulation. Proposed: Proposed method. 


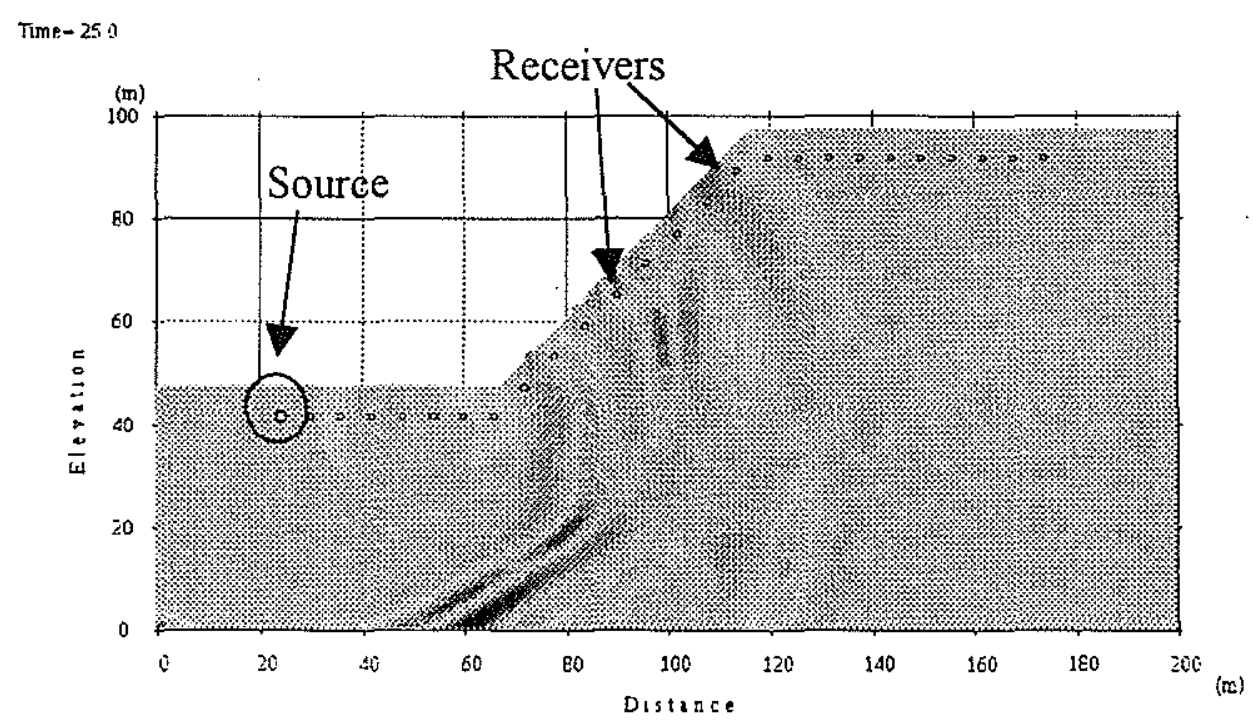

(a) Model A. Grid size $=0.5 \mathrm{~m}$, Step size $=0.5 \mathrm{~m}$

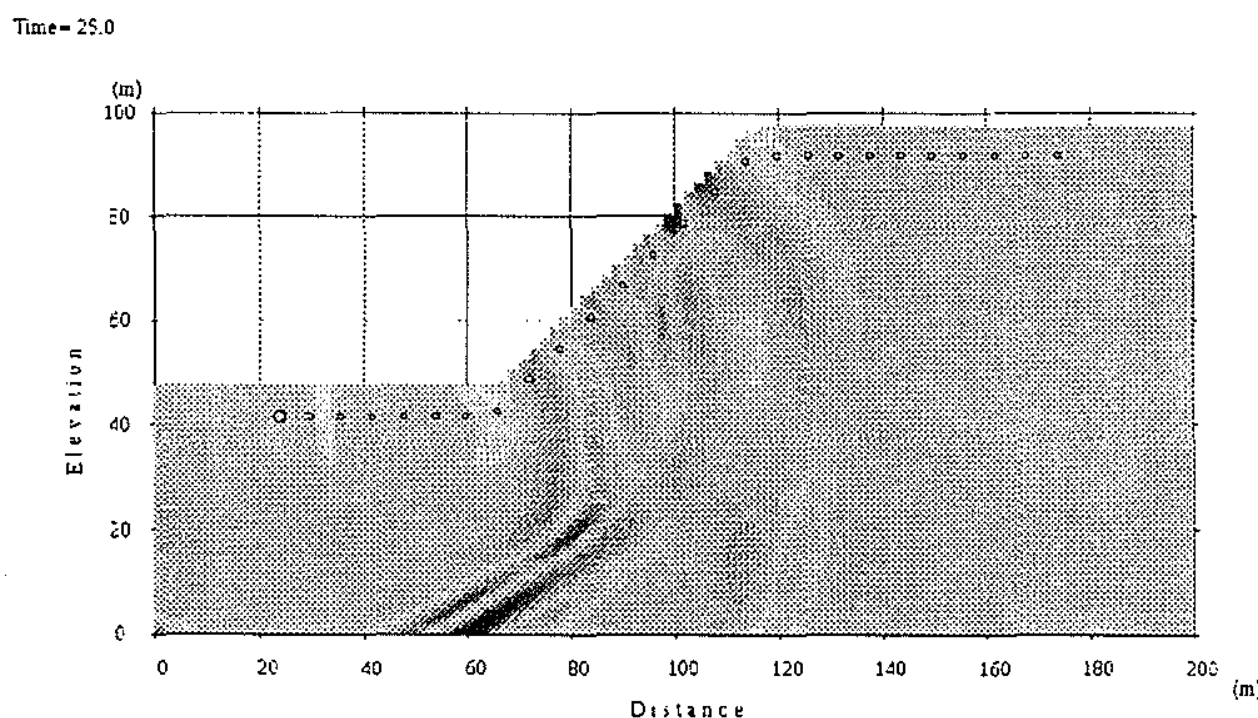

(b) Model F. Grid size $=0.5 \mathrm{~m}$, Step size $=2.0 \mathrm{~m}$

Figure 7: Two models from Table 2 used in the numerical test, with a wavefield of particle velocity (vertical component) at time $25 \mathrm{msec}$. P-velocity $=5082 \mathrm{~m} / \mathrm{s}$; S-velocity $=3000 \mathrm{~m} / \mathrm{s}$; density $=2000 \mathrm{~kg} / \mathrm{m}^{3}$. 
Hayashi and Burns

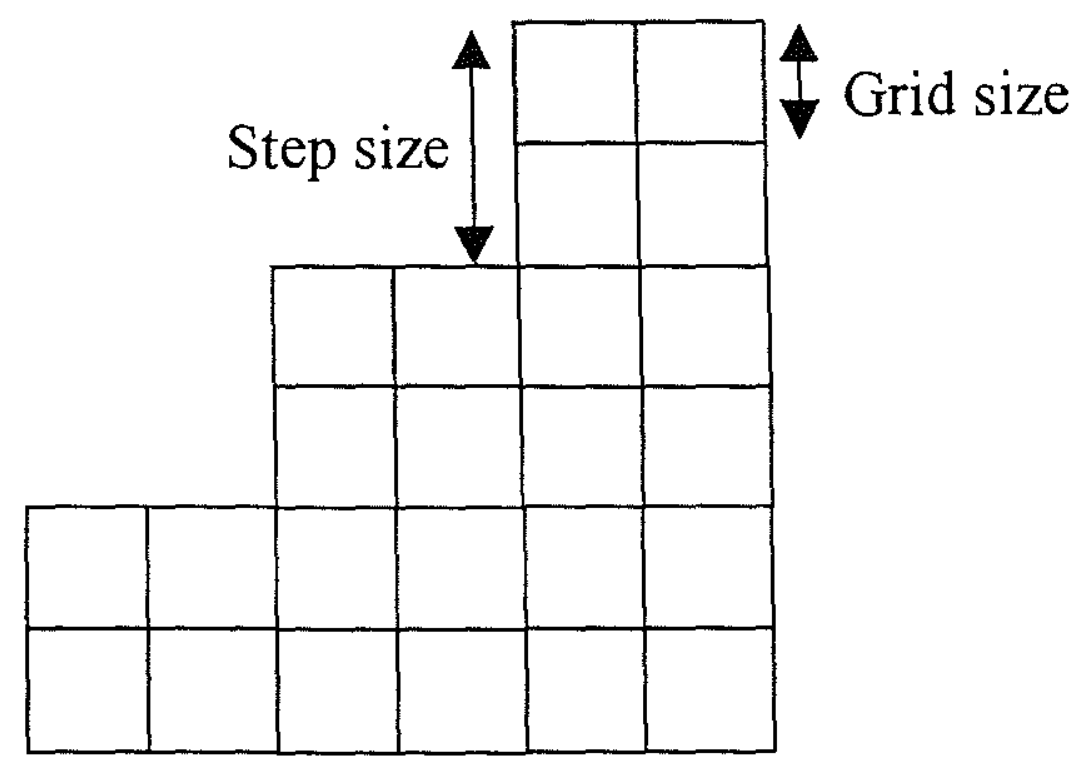

Figure 8: Definition of the grid size and the step size. 
Variable Grid Finite-Difference Modeling

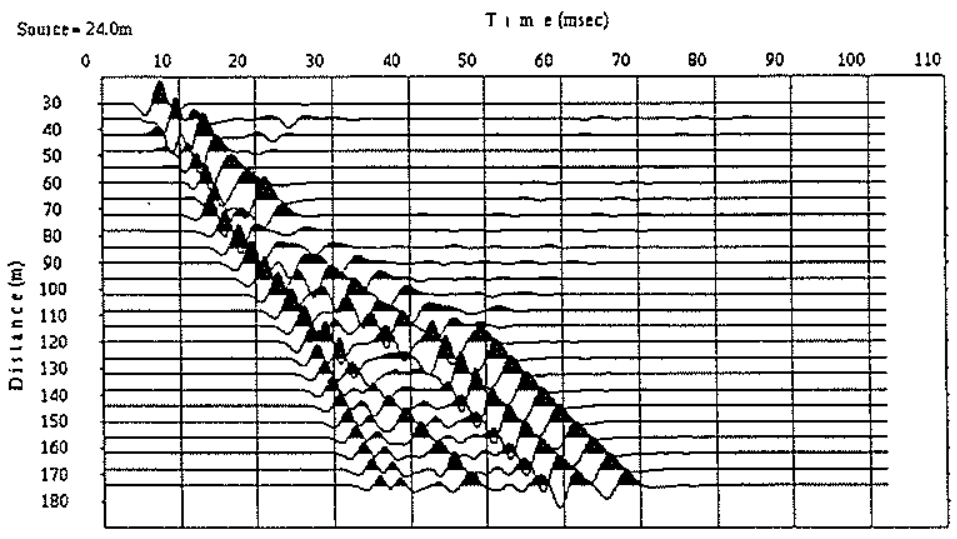

(a) Model A. Grid size $=0.5 \mathrm{~m}$, Stepsize $=0.5 \mathrm{~m}$

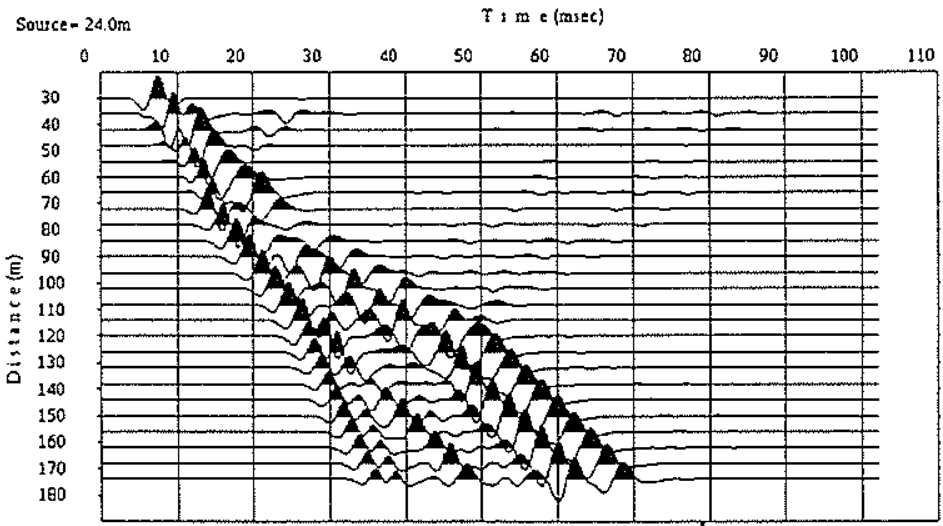

(b) Model B. Grid size $=0.5 \mathrm{~m}$, Step size $=1 \mathrm{~m}$

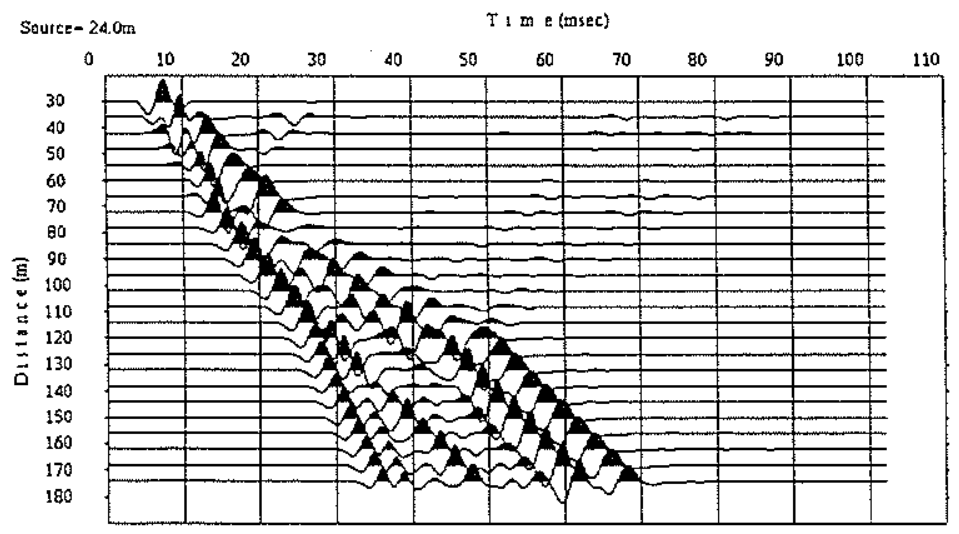

(c) Model C. Grid size $=1.0 \mathrm{~m}$, Step size $=1.0 \mathrm{~m}$

Figure 9: Particle velocity (vertical component) from the simulation with the various grid and step sizes for the models given in Table 2. 
Hayashi and Burns

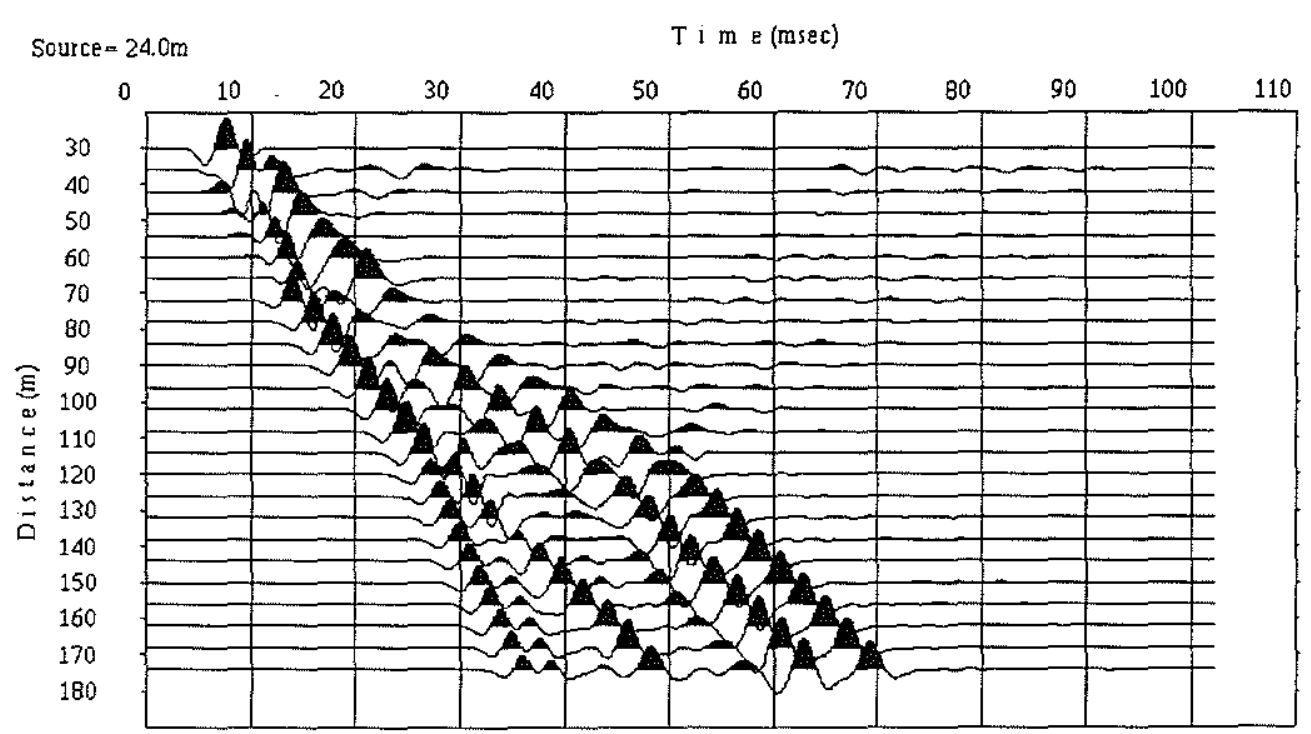

(d) Model D. Grid size $=0.5 \mathrm{~m}$, Step size $=1.5 \mathrm{~m}$

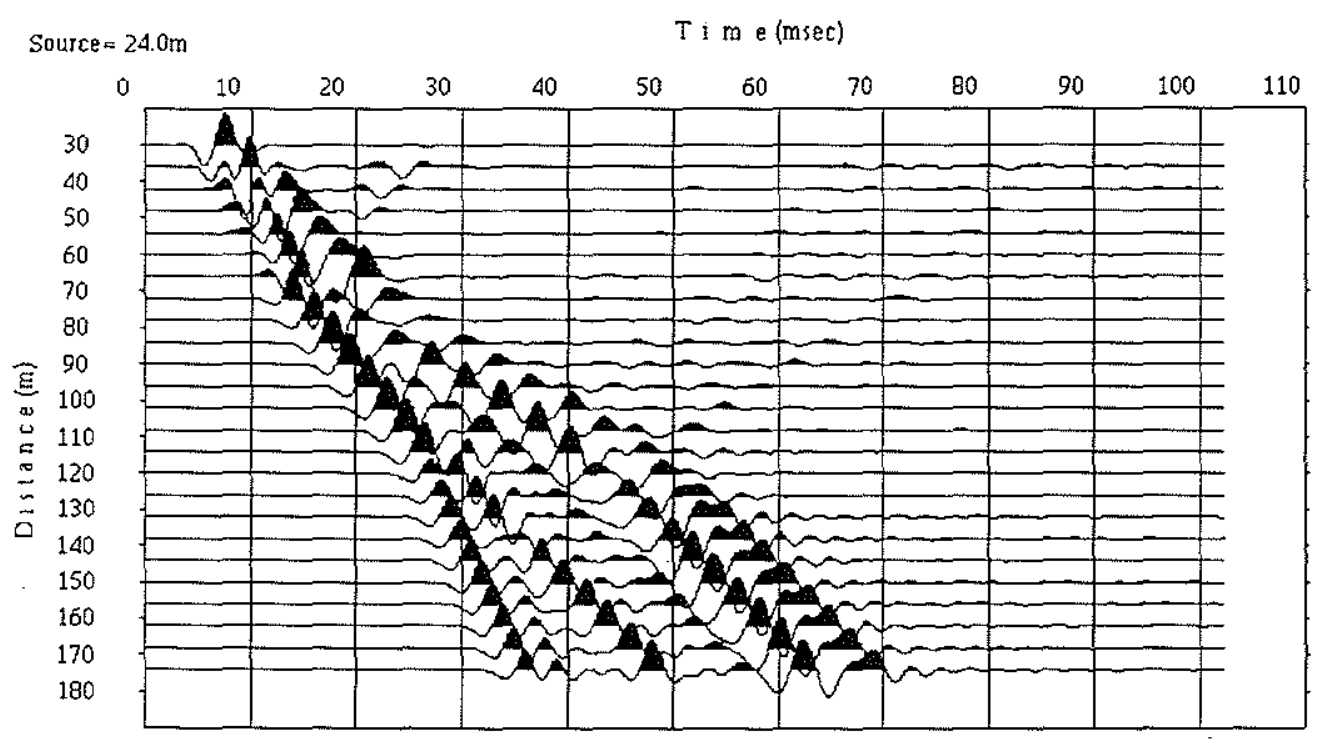

(e) Model E. Grid size $=1.5 \mathrm{~m}$, Step size $=1.5 \mathrm{~m}$

Figure 9, continued: 
Variable Grid Finite-Difference Modeling

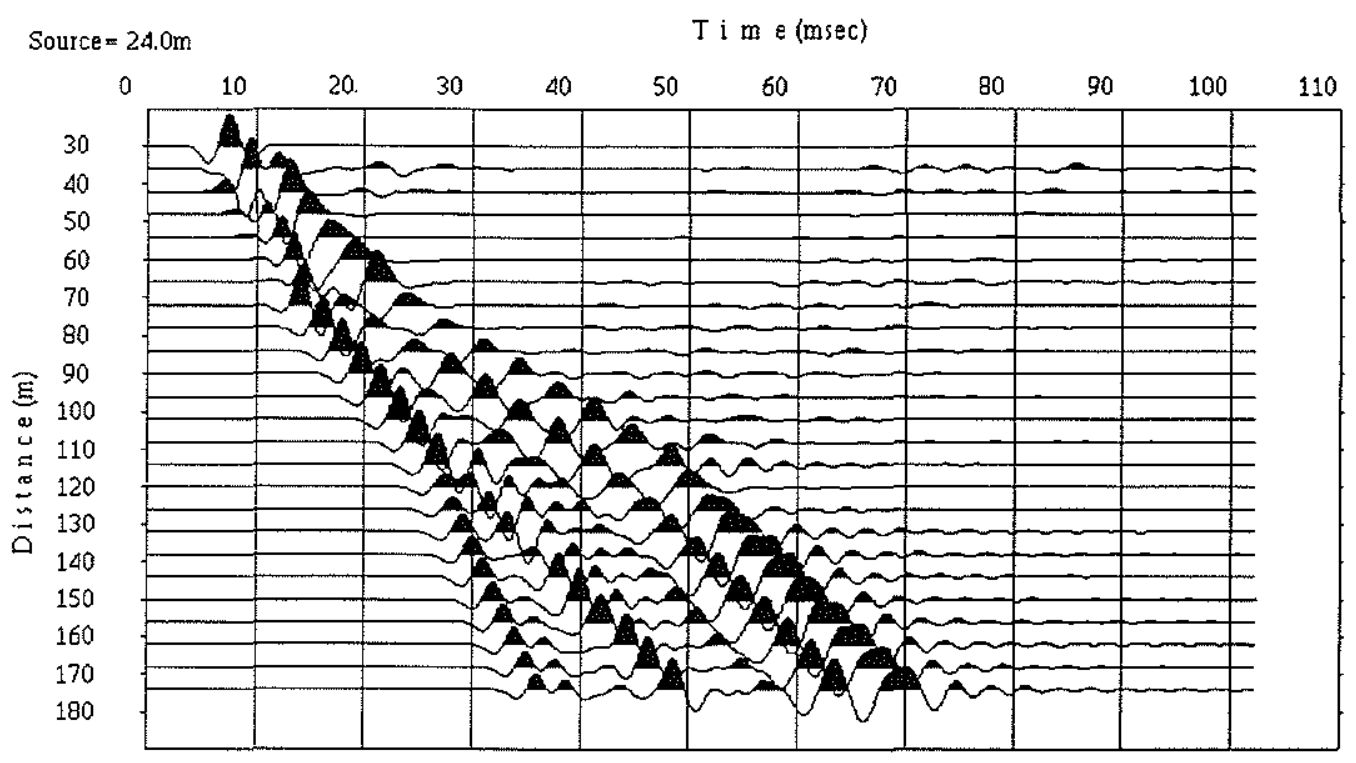

(f) Model F. Grid size $=0.5 \mathrm{~m}$, Step size $=2.0 \mathrm{~m}$

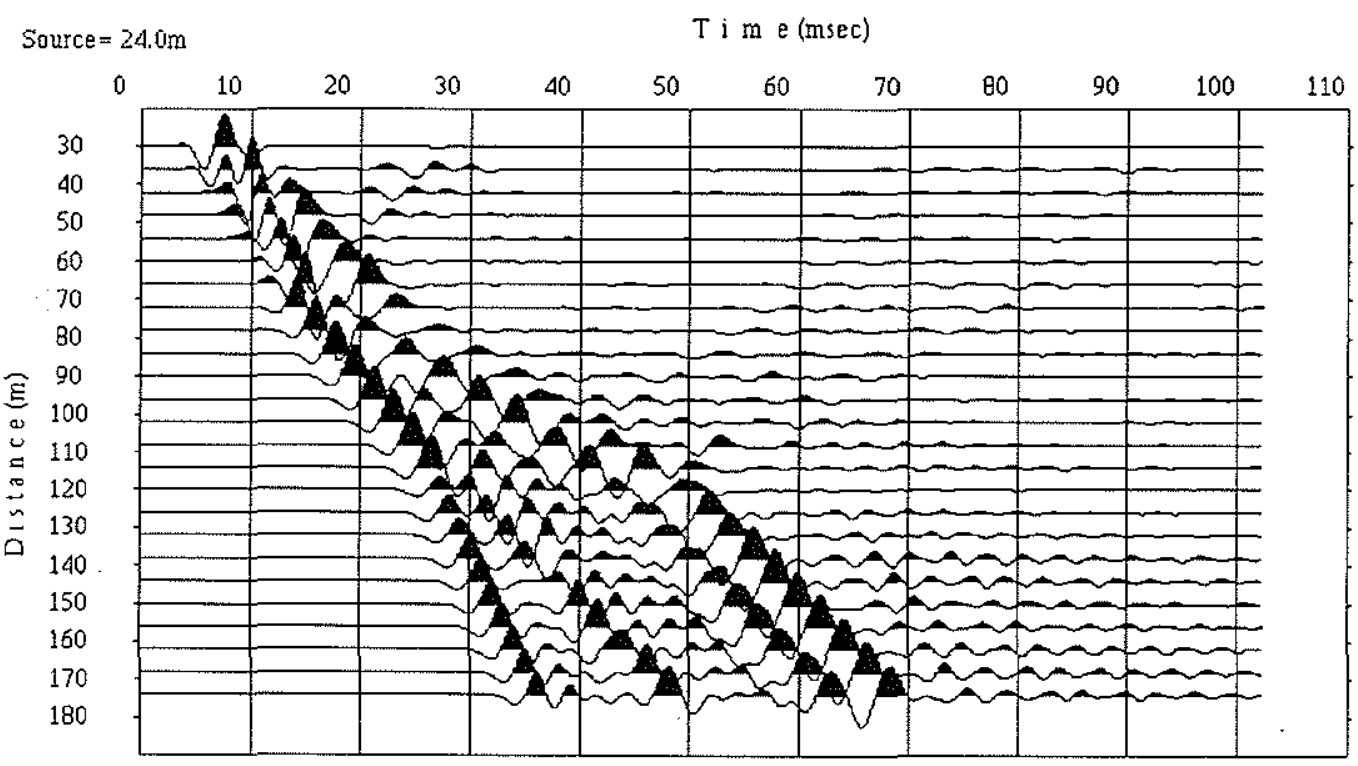

(g) Model G. Grid size $=2.0 \mathrm{~m}$, Step size $=2.0 \mathrm{~m}$

Figure 9, continued: 
Hayashi and Burns

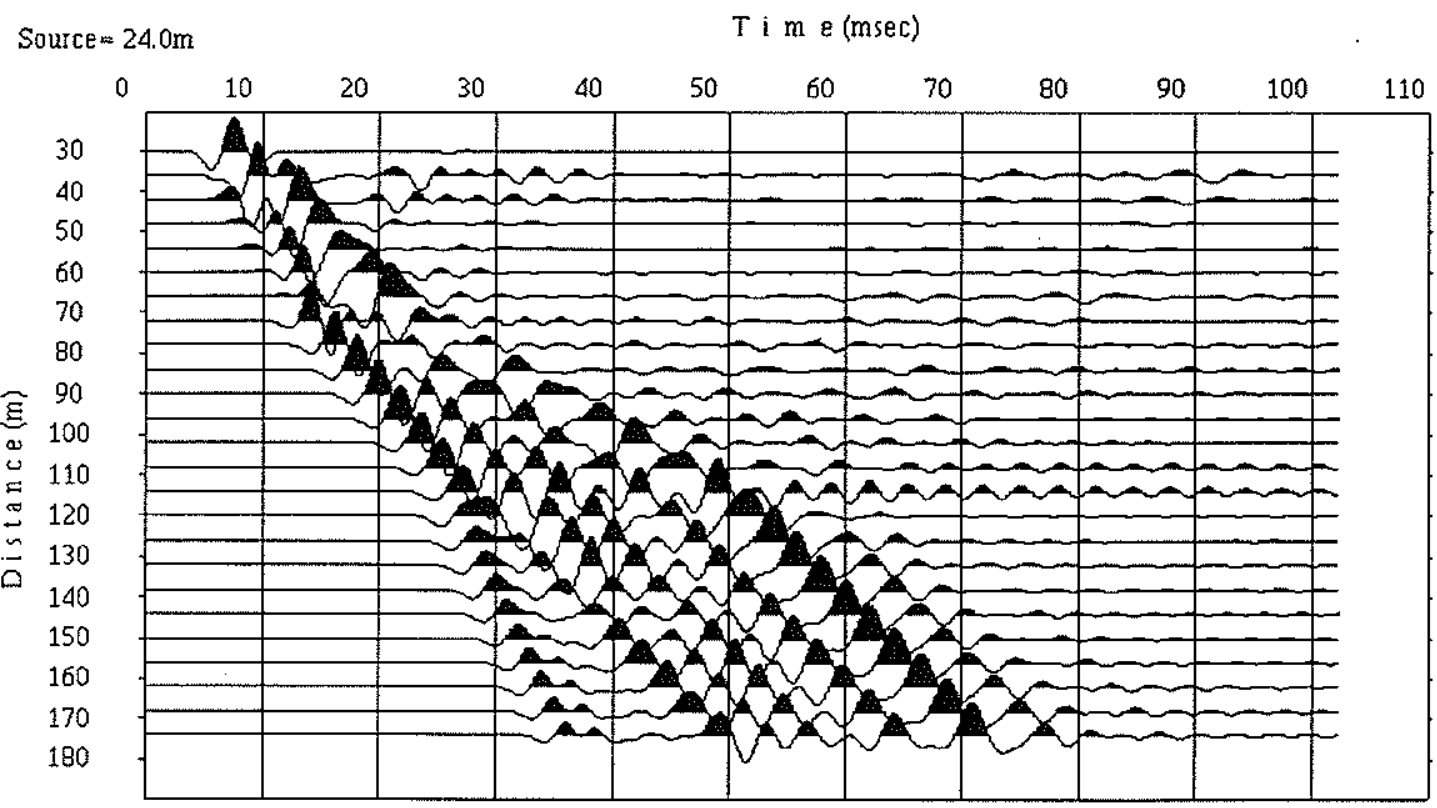

(h) Model H. Grid size $=0.5 \mathrm{~m}$, Step size $=3.0 \mathrm{~m}$

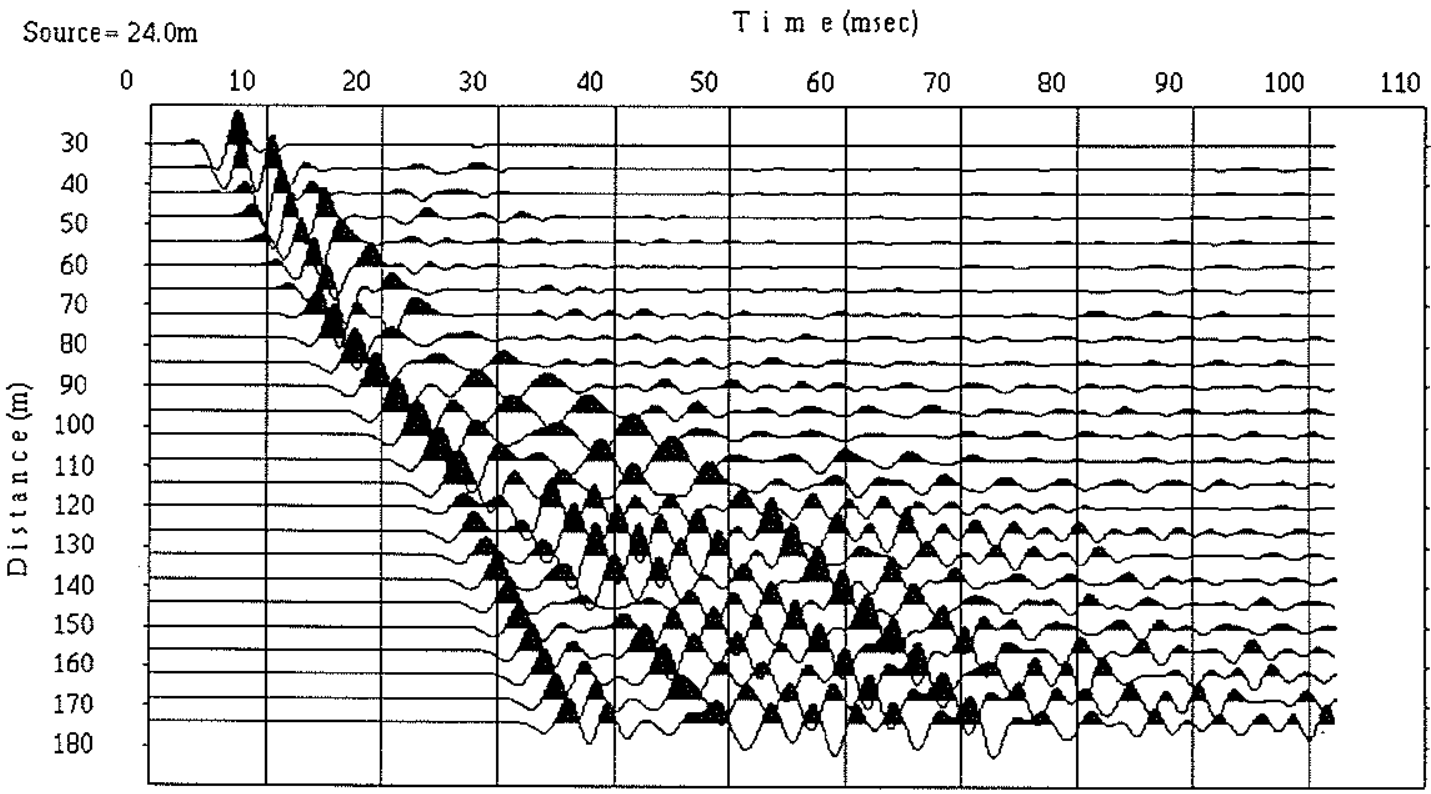

(i) Model I. Grid size $=3.0 \mathrm{~m}$, Step size $=3.0 \mathrm{~m}$

Figure 9, continued: 


\section{Variable Grid Finite-Difference Modeling}

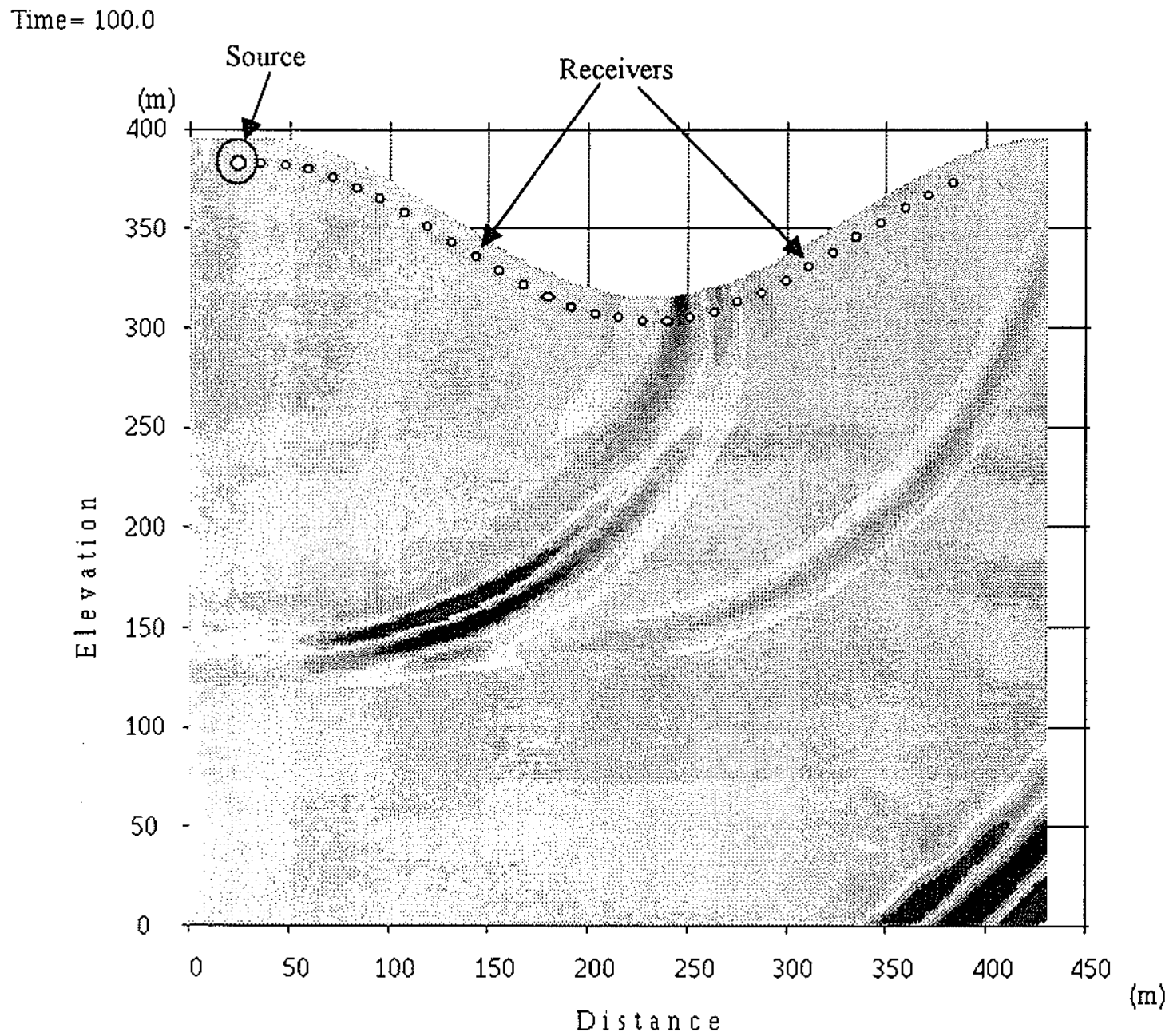

Figure 10: Model used in the numerical test, with a wavefield of particle velocity (vertical component) at time $100 \mathrm{msec}$. P-velocity $=6000 \mathrm{~m} / \mathrm{s} ;$ S-velocity $=3000 \mathrm{~m} / \mathrm{s} ;$ density $=1800 \mathrm{~kg} / \mathrm{m}^{3}$. 
Hayashi and Burns

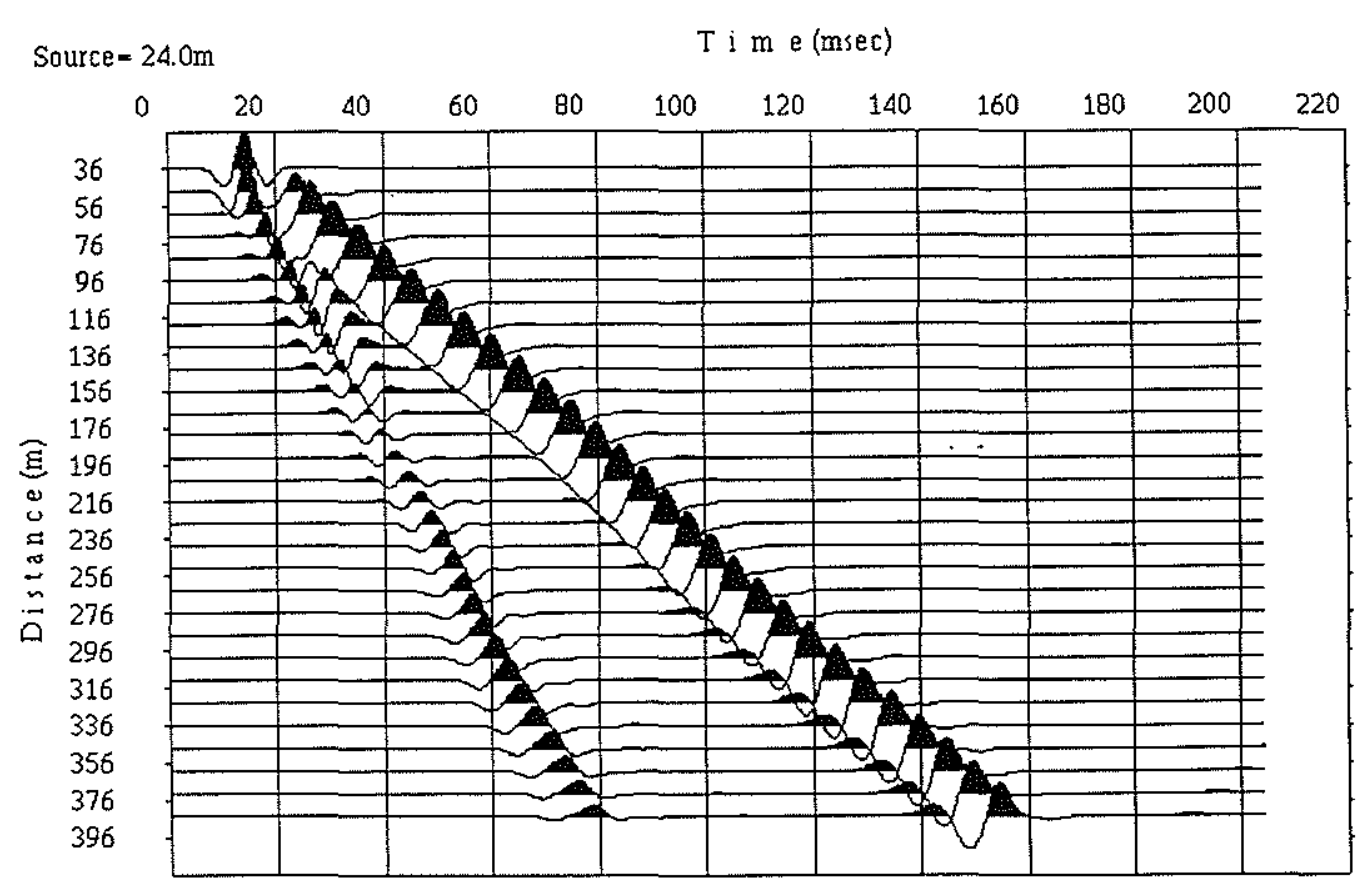

(a) Grid size $=0.5 \mathrm{~m}(\lambda / \Delta \mathrm{x}=60)$

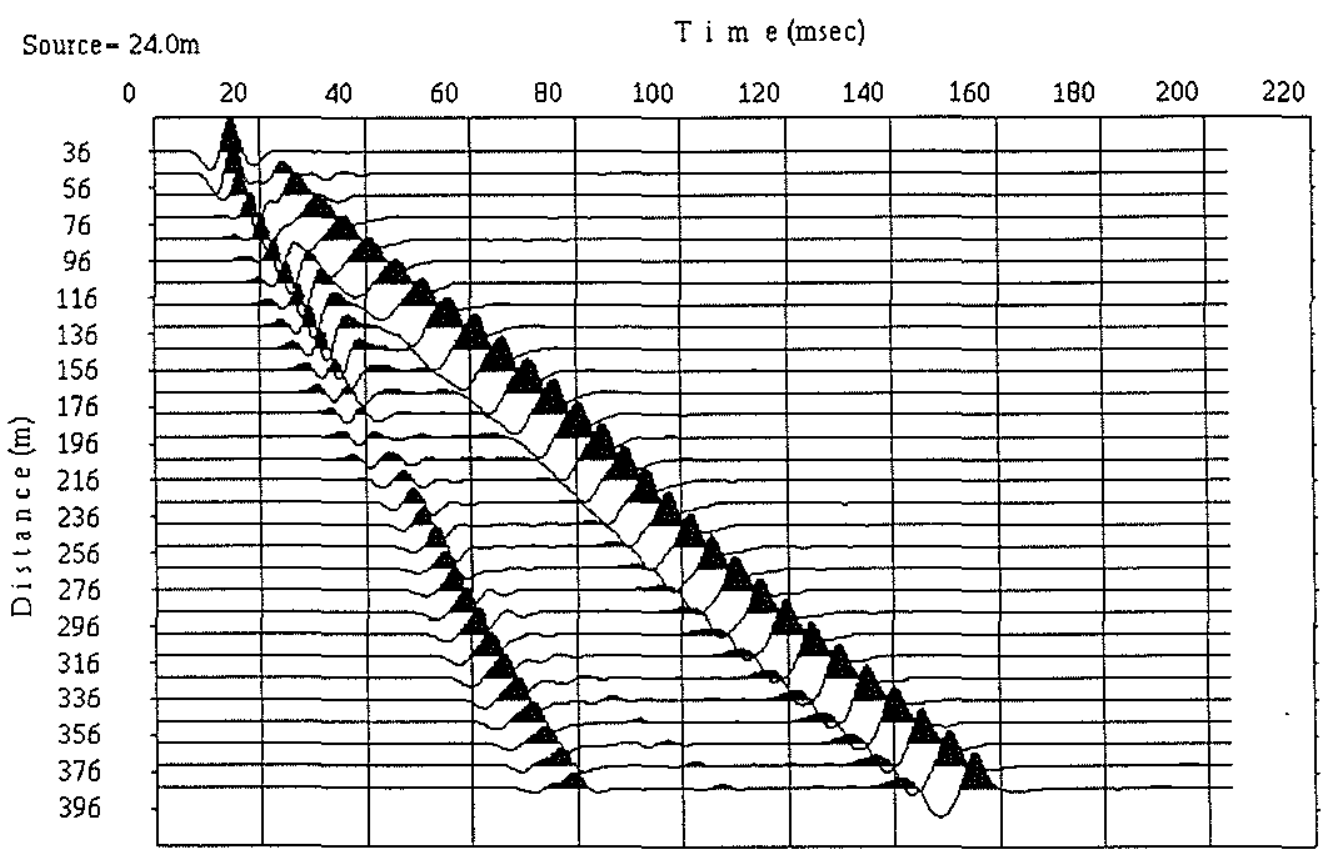

(b) Grid size $=1.0 \mathrm{~m}(\lambda / \Delta \mathrm{x}=30)$

Figure 11: Particle velocity (vertical component) from the simulation with the various grid sizes for the model in Figure 10. 
Variable Grid Finite-Difference Modeling

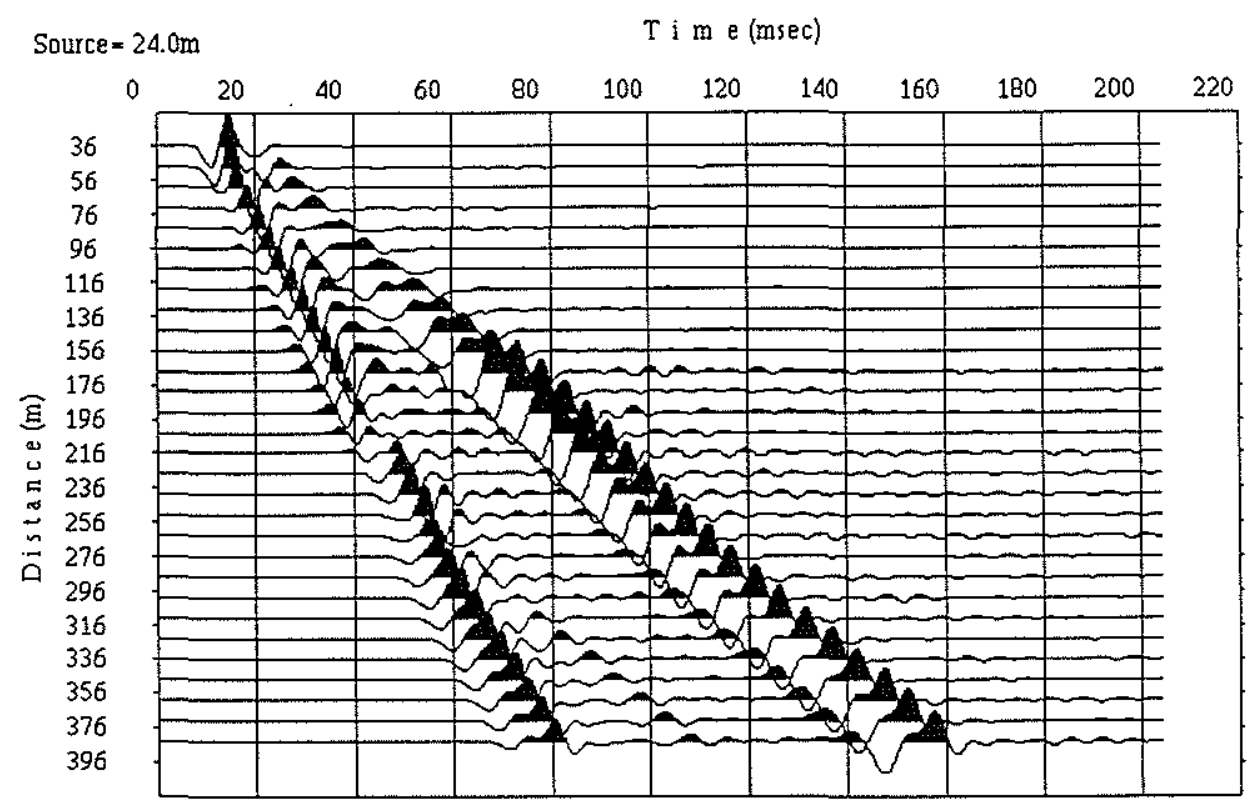

(c) Grid size $=2.0 \mathrm{~m}(\lambda / \Delta \mathrm{x}=15)$

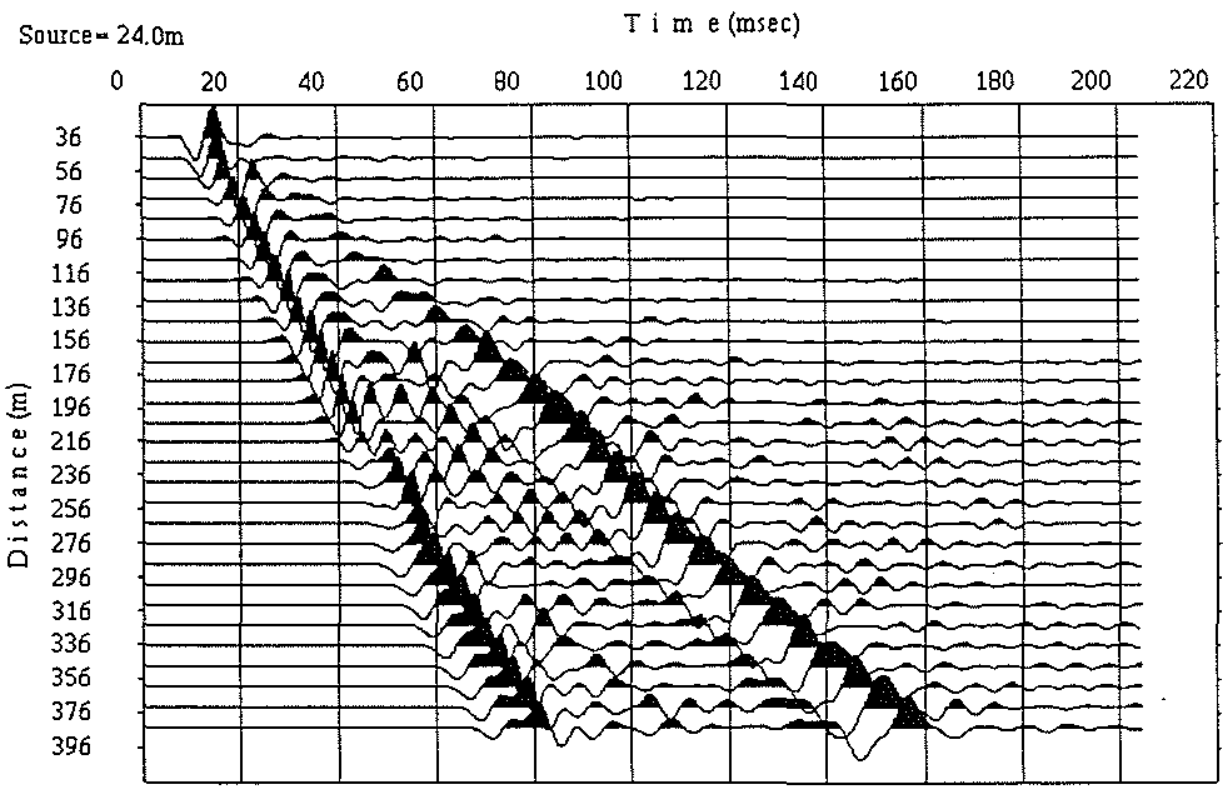

(d) Grid size $=3.0 \mathrm{~m}(\lambda / \Delta \mathrm{x}=10)$

Figure 11, continued: 
Hayashi and Burns

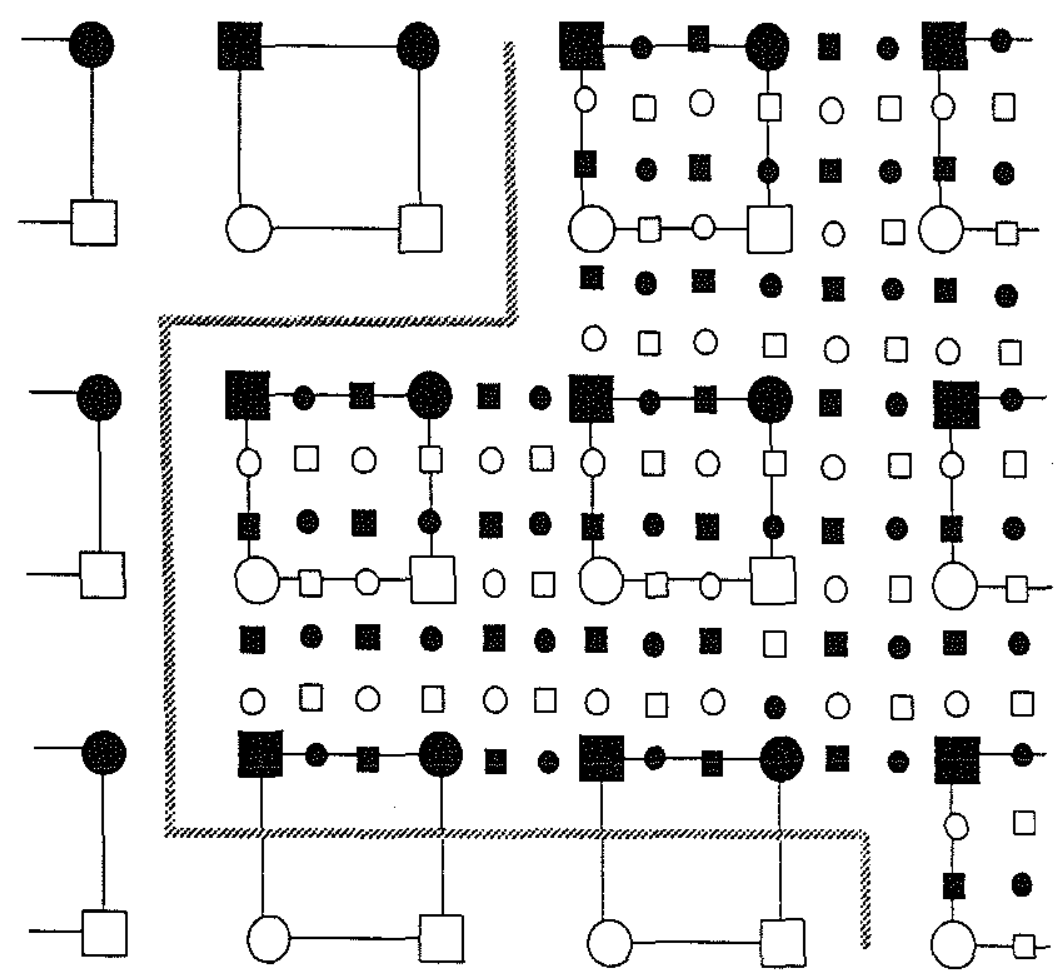

Figure 12: Staggered finite-difference grid in the vicinity of the boundary between different grid sizes. 


\section{Variable Grid Finite-Difference Modeling}
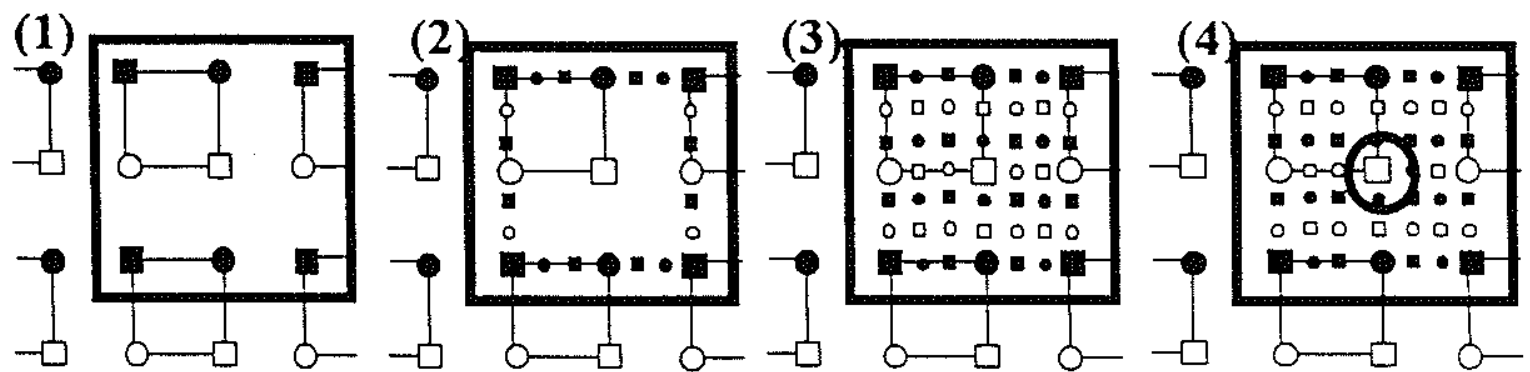

Figure 13: Computation procedure for the variable grid method. (1) Update the coarse

grid. (2) The fine grid at the boundary is interpolated by the coarse grid. (3) Update the fine grid without the boundary. (4) Coarse grid components within the fine grid are replaced by the fine grid. 


\section{Hayashi and Burns}

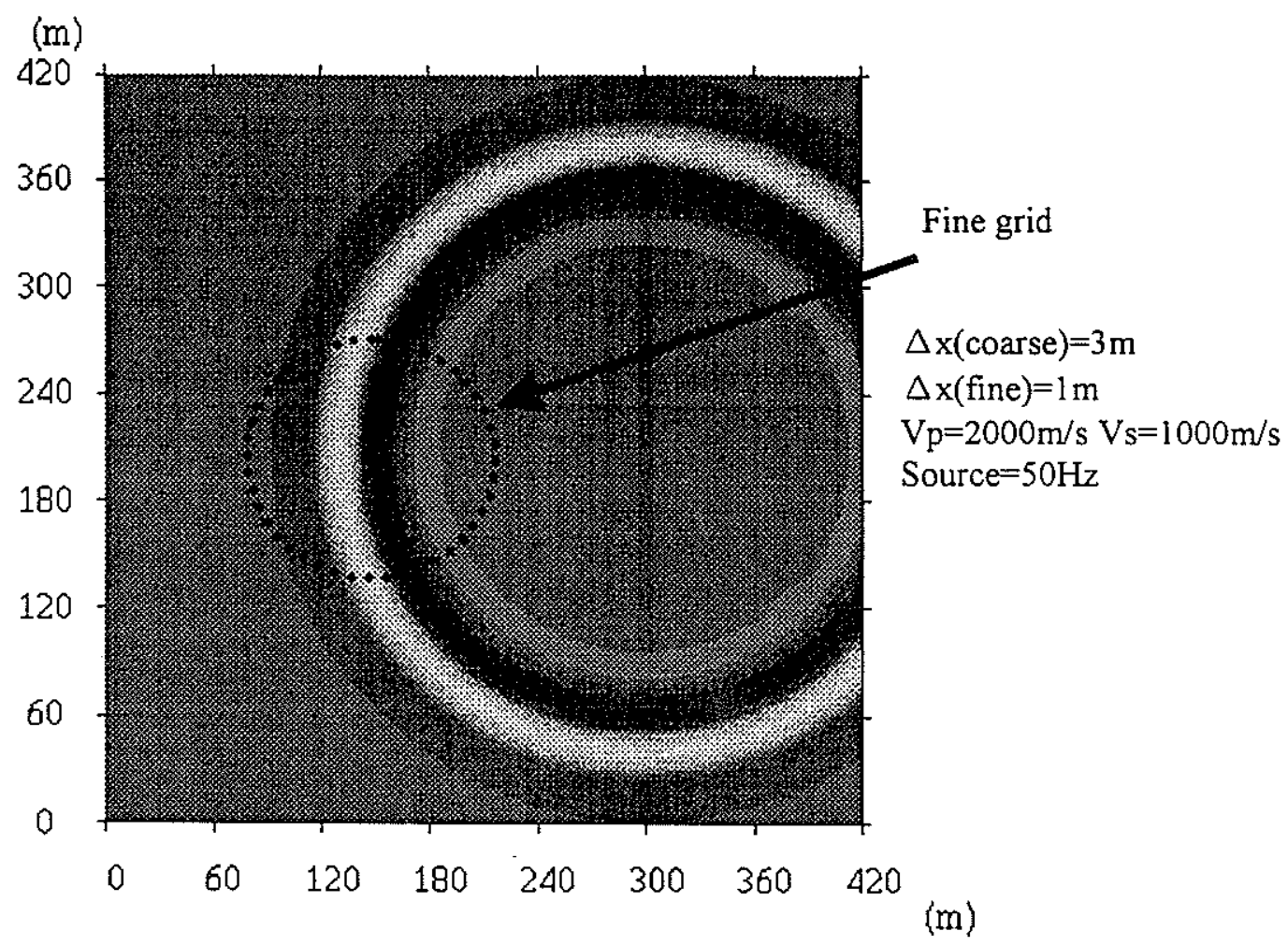

Figure 14: Wavefield snapshot across a variable grid zone (homogeneous case). 
Variable Grid Finite-Difference Modeling

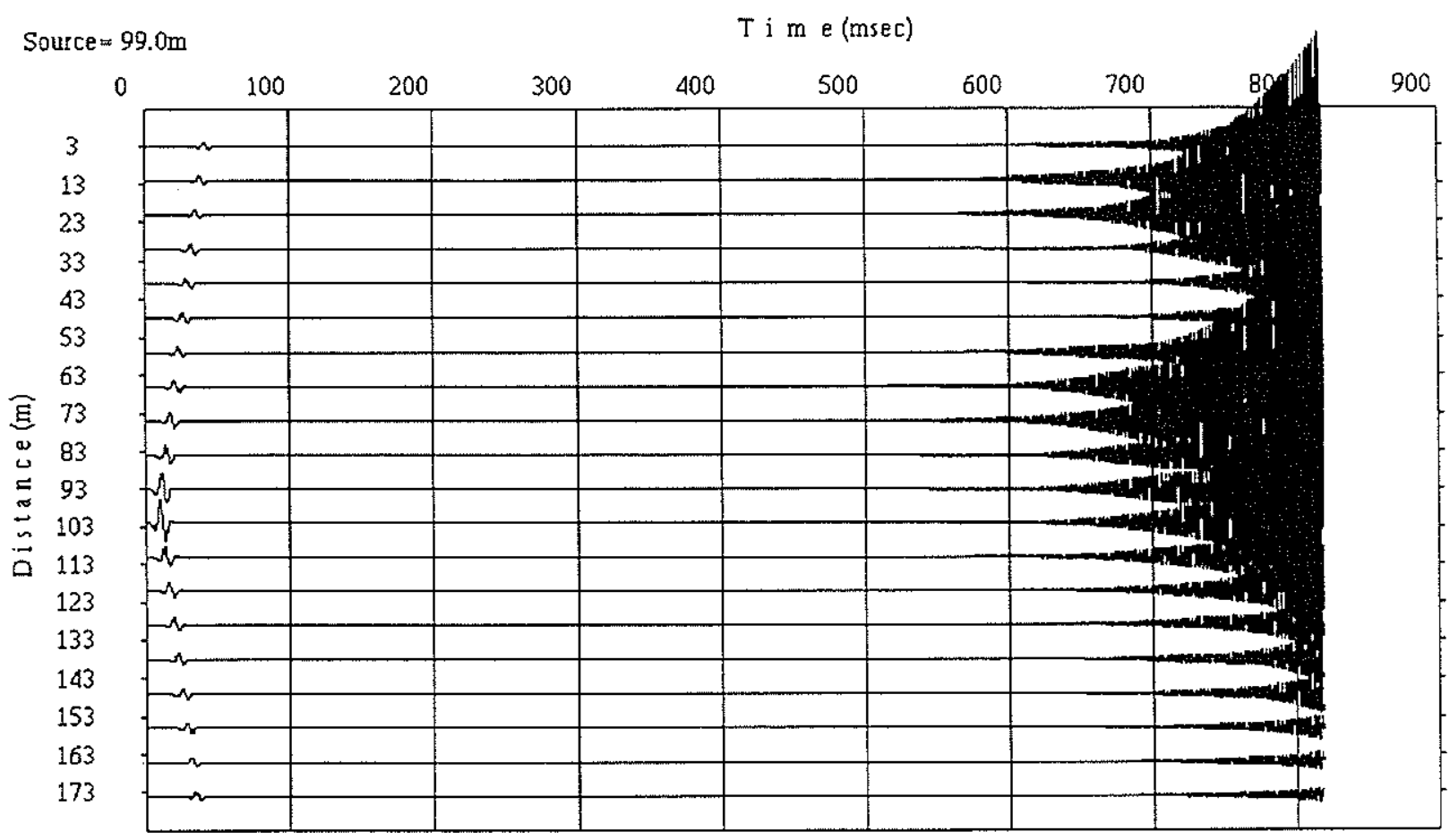

Figure 15: Instability due to the variable grid implementation. 


\section{Hayashi and Burns}

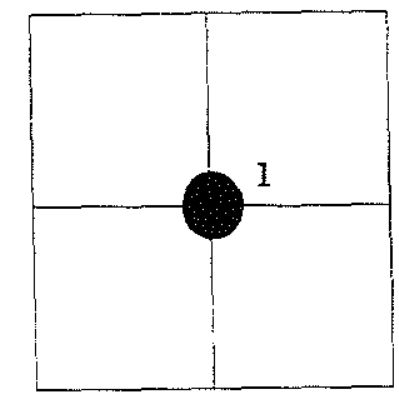

A:No weighting or averaging

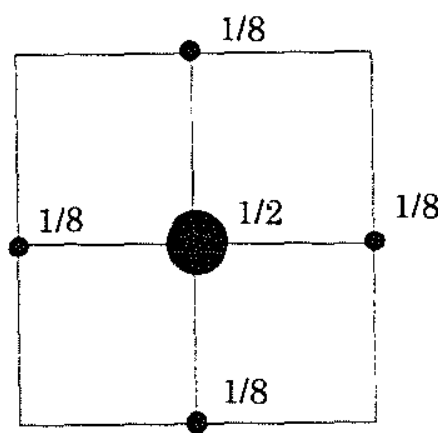

D:5 point weighting

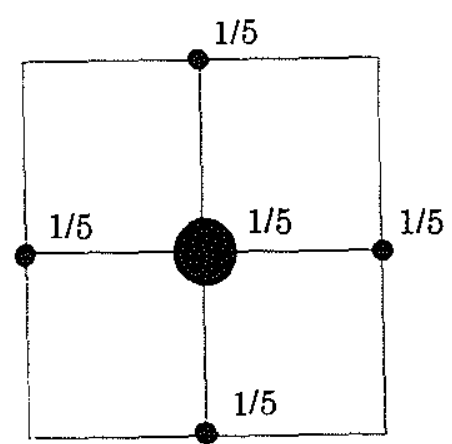

B:5 point averaging

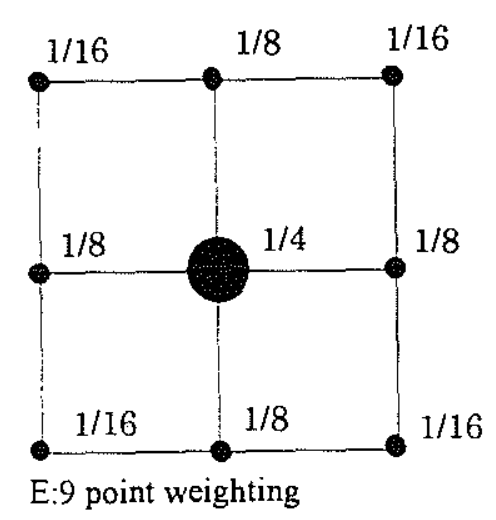

E:9 point weighting

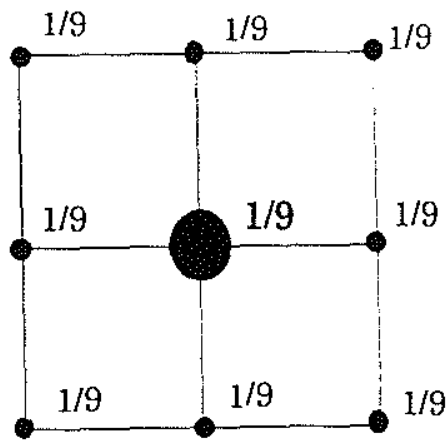

C:9 point averaging

Figure 16: Method of averaging and weighting applied to the replacement of the coarse grid components within the fine grid (Figure 13. Step 4). 


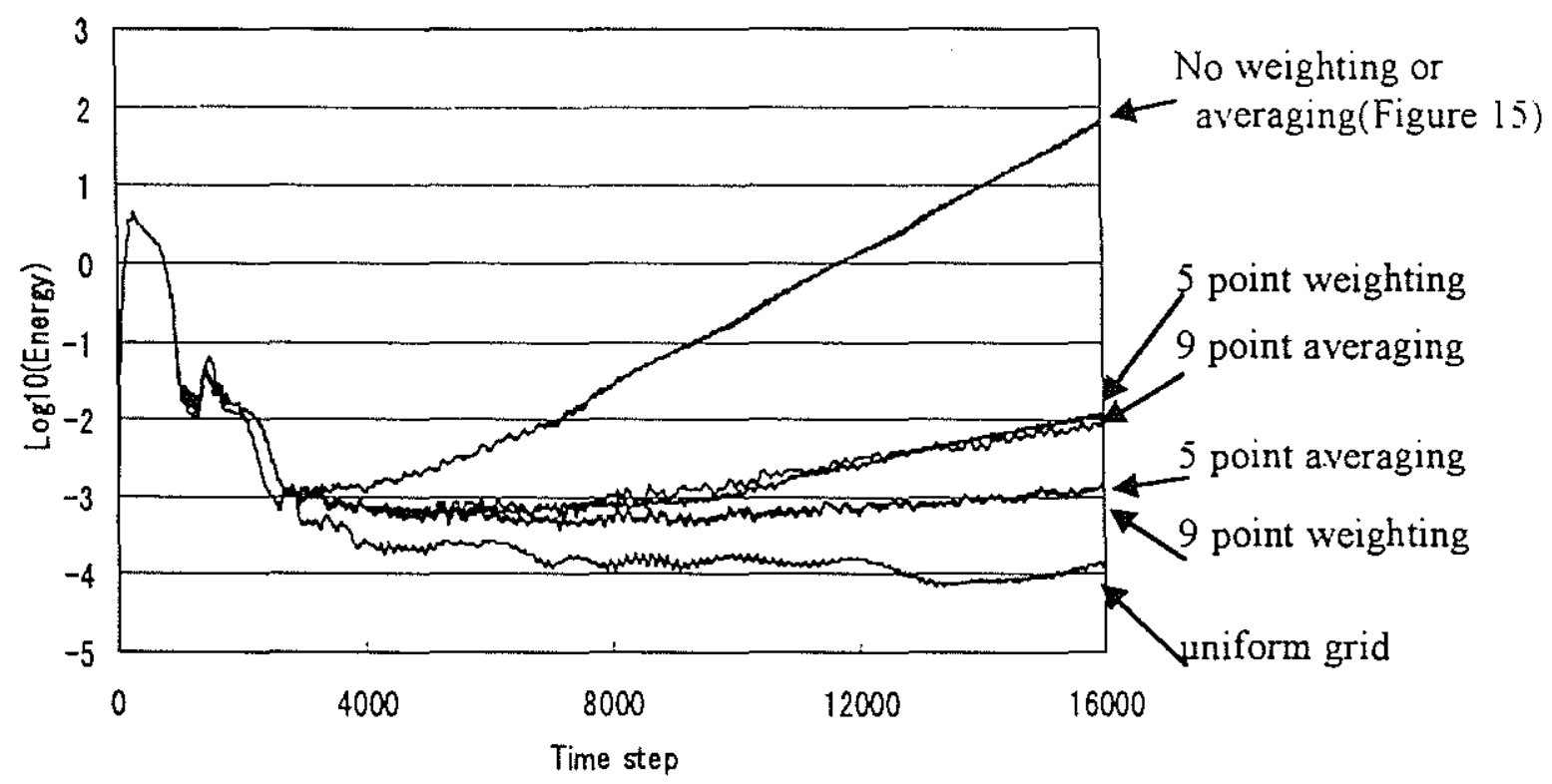

Figure 17: Energy of waveforms shown in Figure 15 with various weighting methods. 


\section{Hayashi and Burns}

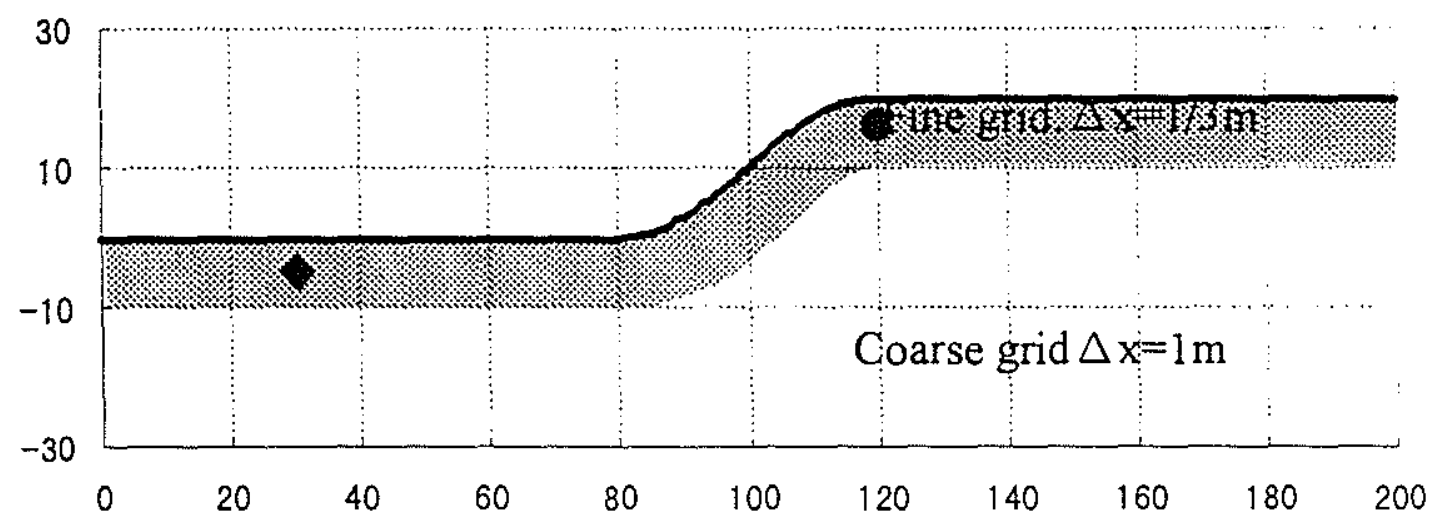

Figure 18: Area of the fine grid. The grid size is $1 / 3 \mathrm{~m}$ in the fine grid, and $1 \mathrm{~m}$ in the coarse grid. 


\section{Variable Grid Finite-Difference Modeling}

T i m e (msec)

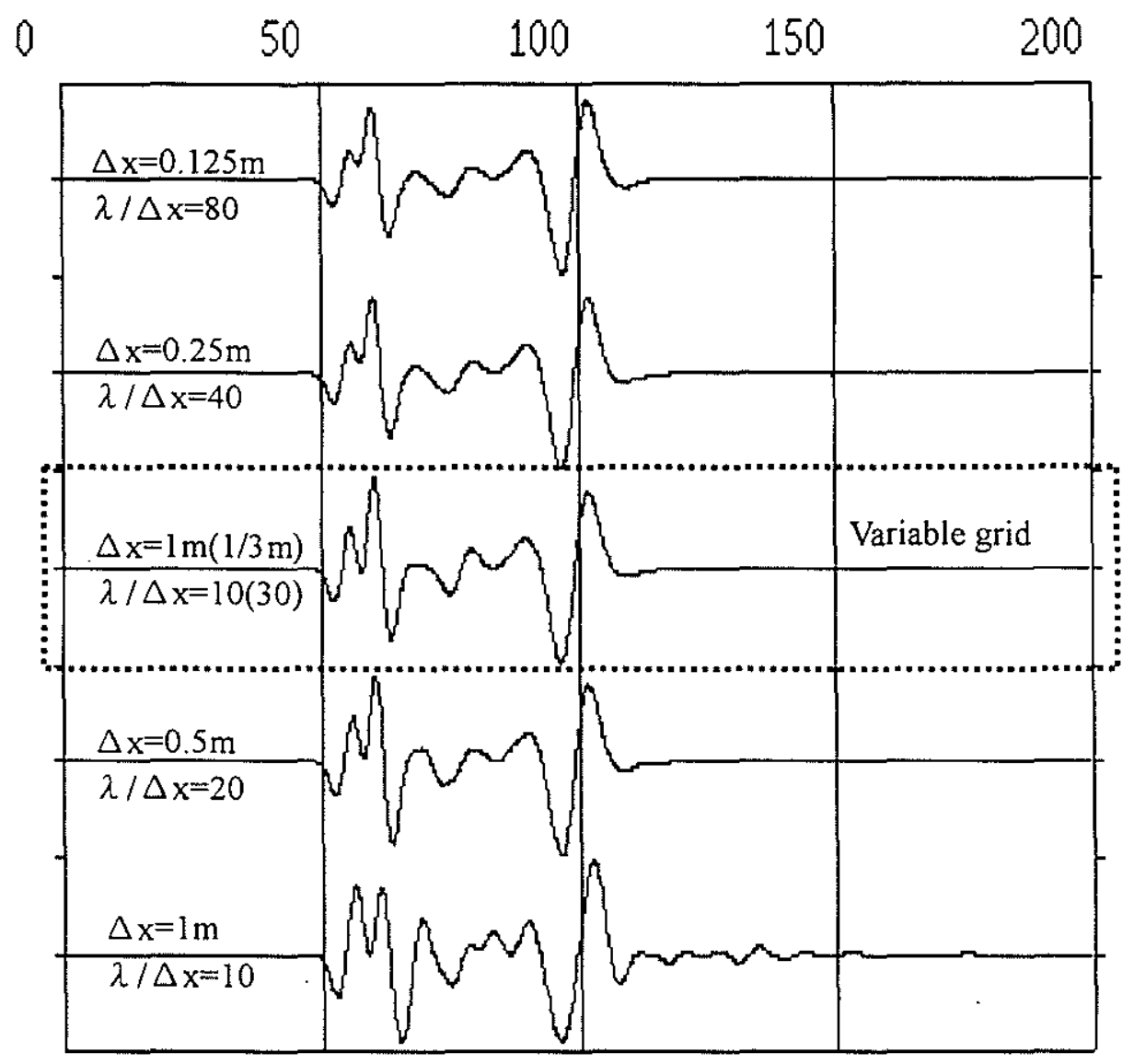

Figure 19: Comparison of waveforms calculated by the proposed method with the various grid sizes. 
Hayashi and Burns

13-36 